\title{
Continuous prescribed-time sliding mode control with a prescribed-time ESO for second-order nonlinear systems with mismatched disturbance
}

\author{
Xiaozhe Ju • Feng Wang • Yingzi Guan • Shihao Xu
}

Received: date / Accepted: date

\begin{abstract}
This paper aims to settle the continuous prescribed-time stabilization problem of secondorder nonlinear systems with mismatched disturbances. A continuous prescribed-time sliding mode control (CPTSMC) method with a prescribed-time extended state observer (PTESO) is proposed. The PTESO can precisely estimate the unknown states and disturbances, with its upper bound for the settling time (UBST) prescribed by only one parameter more tightly than existing finite-time or fixed-time ESOs. Furthermore, as a common concern for ESOs, the peaking value problem is well addressed. Then, a novel prescribedtime convergent form with little conservatism and simple tuning procedures is designed, and the internal mechanism in acquiring higher transient performance is explicitly researched. By using the estimated states and disturbances, the CPTSMC makes system states converge in a chattering-alleviated manner following the novel prescribed-time form. In addition to proving that the UBST of the whole system is tightly prescribed by only one design parameter, we show the continuity of the CPTSMC and the boundedness of all system signals, which are vital for practical applications. Ultimately, numerical simulations on the second-order system and a DC motor servo verify the efficiency of the proposed control system.
\end{abstract}

Keywords mismatched disturbances · upper bound for the settling time $\cdot$ continuous prescribed-time sliding mode controller $\cdot$ prescribed-time extended state observer $\cdot$ novel prescribed-time convergent form

Xiaozhe Ju · Feng Wang · Yingzi Guan · Shihao Xu

School of Astronautics, Harbin Institute of Technology,

Harbin 150001, China

E-mail: guanyz@hit.edu.cn

\section{Introduction}

For nonlinear practical application scenarios with timely stabilization and disturbance rejection demands, such as missile interception guidance $[1,2]$, fault detection and recovery $[3,4]$, and attitude orientation of spacecraft $[5,6]$, settling time and disturbance-rejection ability are two important performance specifications of a control system. As a result of this, the finite-time sliding mode control (FTSMC) and fixed-time sliding mode control (FxTSMC), known for their fast convergence rate and strong robustness against disturbance, have been appealing in recent decades [7]. Many efforts have been made to develop novel FTSMC and FxTSMC, including Lyapunov-based SMC [7-10] and homogeneitybased SMC [11,12]. However, as analyzed in [13], the Lyapunov-based FTSMC/FxTSMC laws often hold a complicated and conservative relationship between multiple design parameters and the estimated upper bound for the settling time (UBST), shown in [7-10]. While for the homogeneity-based methods, the finite-/fixed-time stability is validated from the asymptotic stability of various homogenous approximations without obtaining an estimate of the UBST [11,12].

The prescribed-time sliding mode control (PTSMC), with the estimated UBST concisely related with design parameters, has attracted increasing interest in overcoming the aforementioned problems of FTSMC/FxTSMC. The existing prescribed-time control laws can be categorized into two varieties. One is based on a kind of Lyapunov-like characterization, which guarantees the actual settling time equal to the prescribed UBST under the condition of infinite initial state errors [14]. Many PTSMC laws have been developed within this framework for Euler-Lagrange systems [15], distributed-order systems [16], under- 
water vehicles [17], robotic manipulators [18], and so on. Nonetheless, regarding the PTSMC [15-18], the conservativeness in estimating UBST has not been effectively reduced, and the actual convergence rate becomes unnecessarily fast for practical scenarios under small initial values. Another variety of prescribed-time control laws is based on time-varying gains and has been presented in [19-26]. The actual settling time of the system is approximate to the prescribed UBST regardless of the magnitude of initial state errors. In other words, UBST can be prescribed in a weakly conservative manner (it is called prescribing the UBST tightly in this paper), which is more suitable for practical applications with a small desired settling time and motivates us to carry out further research on this variety. At present, there have been few attempts to develop the PTSMC using time-varying gains. Anil Kumar Pal et al. proposed an integral PTSMC $[27,28]$ to drive integrator systems to the equilibrium point within the prescribed time interval, but the boundedness of the control efforts was not proved. Lei Cui et al. derived a time-varying PTSMC for partial integrated guidance and control [29]. Z Chen developed a time-varying PTSMC for the attitude tracking of spacecraft [30]. However, the PTSMC laws in [27-30] are discontinuous. Consequently, severe chattering may occur, considering the finite sampling frequency of practical applications. Therefore, the utility of the time-varying continuous PTSMC (CPTSMC) remains an open issue and is chosen as the research topic of this paper to alleviate chattering in practice.

Most importantly, although there exist some timevarying prescribed-time control methods for the highorder integrator systems [19], p-norm nonlinear systems [21], and strict-feedback-like nonlinear systems [23], the research [19-30] has not coped with nonlinear systems with mismatched state-independent disturbance, which are commonly encountered in practice, such as hypersonic vehicles [31], vertical take-off and landing reusable launch vehicles [32], active suspension systems [33], and DC motor servos [34,35]. Considering the fast convergence demands and the existence of mismatched stateindependent disturbances, designing a novel CPTSMC with little conservativeness for temporal demands is both meaningful and challenging.

As revealed in [36], when integrated with the baseline robust control law, the extended state observer (ESO) is an effective tool to address both mismatched and matched disturbances by transforming them into lumped disturbances. A typical linear ESO reconstructs the unknown system states and disturbance with asymptotically convergent observation errors. High gains have been employed to accelerate the reconstruction rate, which was accompanied by large peak observation errors (i.e., the peaking value problem) [37]. After the evolution from linear ESOs to nonlinear ESOs, fractional power functions were utilized for fast observation with lowered peaking values. Lyapunov-/homogeneity-based finite-/fixed-time nonlinear ESOs were extensively researched, as seen in [38-41]. Similar to the FTSMC and FxTSMC, the complicated relationship between the estimated UBST and the design parameters, and the over-conservative estimate of UBST, are two main deficiencies for existing nonlinear ESOs. At present, few attempted to develop a prescribed-time extended state observer (PTESO). Rodrigo Aldana-Lopez et al. [42] and Seeber et al. [43] realized the prescribed-time stability (PTS) of robust exact differentiators, which are used to observe the first-order derivative of time-varying signals but cannot be treated as an ESO. Two prescribed-time state observers were proposed in $[44,45]$ for unperturbed systems. The only existing PTESO for time-varying disturbances was recently given in [29]. Hence, it is still of great interest to design a novel PTESO with the UBST prescribed by one parameter tightly. Moreover, it will be more favourable if the PTESO can avoid large peak observation errors at the same time of quick observation.

Motivated by the previous investigation and analysis, we propose a PTESO-based CPTSMC for secondorder nonlinear systems with mismatched disturbances. The closest work to our approach is the latest outstanding research in [29], which also comprises a PTESO and PTSMC. It is noteworthy that our control method is superior to the work of [29] in six aspects. First, the research object is extended from a second-order integrator system to a second-order system with mismatched disturbance, which is more general in practical applications. Second, the proposed PTESO enjoys more straightforward tuning rules than the PTESO in [29]. Third, extending the proposed PTESO to a high-order PTESO is convenient and trivially increases the complexity of tuning parameters. In contrast, with the order increase of the PTESO in [29], the complexity of tuning procedures increases rapidly. Fourth, the proposed CPTSMC is continuous, causing less chattering than the PTSMC in [29]. Fifth, when adjusting the UBST online, only two parameters of our PTESO and CPTSMC need to be tuned. However, six parameters and a linear matrix inequality should be calculated in [29]. Finally, as the time approaches the prescribed UBST, the gains of the PTSMC and PTESO in [29] grow much faster than those of our CPTSMC and PTESO, unwelcomed by practical applications with limited memory space and finite sampling frequency.

The main contributions are summarized here: 
1. A time-varying continuous prescribed-time controller for a second-order nonlinear system with mismatched disturbances is researched. To the best of our knowledge, this has not been addressed by the existing literature (such as [19-30]).

2. A PTESO is derived to reconstruct unknown system states and disturbances, with the UBST of observation errors prescribed by one design parameter. Compared to other ESOs [36-41], the parameter tuning of the PTESO for temporal demands is simpler and less conservative. Furthermore, large peak observation errors are efficiently avoided.

3. A novel prescribed-time convergent form is developed by adding a power term, of which the internal mechanism in adjusting the transient performance is explicitly analysed. Accordingly, a CPTSMC is derived based on the novel convergent form, enabling the UBST of system states prescribed by only one parameter. Chattering is alleviated compared to the PTSMC [15-18,27-30], and conservativeness in meeting temporal demands is reduced compared to the PTSMC in [15-18]. Moreover, the boundedness of all system signals has been verified.

The remainder is arranged as follows. The control problem is stated in Section 2. The design process of the PTESO and CPTSMC is detailed in Section 3, together with the rigorous PTS proof of the whole system. Section 4 presents simulation results and analysis. Conclusions are given in Section 5.

Notations This paper uses $\mathbb{R}, \mathbb{R}_{+}$and $\mathbb{R}_{>0}$ for the set of real numbers, positive real numbers and nonnegative real numbers, respectively. $t_{0}$ denotes the initial time. For $x \in \mathbb{R},\lceil x\rfloor^{\alpha}=|x|^{\alpha} \operatorname{sign}(x)$ with $\alpha \in \mathbb{R}_{\geq 0}$ and $\operatorname{sign}(x)$ as the sign function. $C^{1}$ function denotes the function owning the continuous first-order derivative. For $\breve{\boldsymbol{x}}=\left[x_{1}, x_{2}, \cdots, x_{n}\right] \in \mathbb{R}^{n}, \frac{\partial f(\breve{\boldsymbol{x}})}{\partial x_{i}}$ means the partial derivative of $f(\breve{\boldsymbol{x}})$ with respect to $x_{i}$.

\section{Problem Statement}

Second-order nonlinear systems with mismatched stateindependent disturbance are common in practical applications, such as in [31-35]. When facing with these systems, the prescribed-time methods [19-30] become inapplicable. Thus, oriented for this kind of nonlinear system, this paper is devoted to exploring a chatteringalleviated prescribed-time control method, which has simple parameter tuning procedures and little conservatism for temporal demands (physically realizable).

Consider the second-order system [46] as follows:

$$
\left\{\begin{array}{l}
\dot{x}_{1}=x_{2}+f_{1}(\boldsymbol{x})+d_{1}(t) \\
\dot{x}_{2}=f_{2}(\boldsymbol{x})+d_{2}(t)+b(\boldsymbol{x}) u
\end{array}\right.
$$

where $\boldsymbol{x}=\left[x_{1}, x_{2}\right]^{\mathrm{T}}$ and $u$ are system states and control input, respectively. $f_{i}(\boldsymbol{x}): \mathbb{R}^{2} \rightarrow \mathbb{R}, i=1,2$ with $f_{1}(\boldsymbol{x})$ as a $C^{1}$ function. $d_{1}(t)$ and $d_{2}(t)$ are bounded unmeasurable disturbances with $d_{1}(t)$ as a $C^{1}$ function.

By using Eq. (1), twice differentiating $x_{1}$ with respect to $t$ yields:

$\ddot{x}_{1}=f_{2}(\boldsymbol{x})+\frac{\partial f_{1}(\boldsymbol{x})}{\partial x_{1}}\left(x_{2}+f_{1}(\boldsymbol{x})\right)+\frac{\partial f_{1}(\boldsymbol{x})}{\partial x_{2}} f_{2}(\boldsymbol{x})$
$+\left(b(\boldsymbol{x})+\frac{\partial f_{1}(\boldsymbol{x})}{\partial x_{2}} b(\boldsymbol{x})\right) u+D(t)$

with $D(t)=d_{2}(t)+\dot{d}_{1}(t)+\frac{\partial f_{1}(\boldsymbol{x})}{\partial x_{1}} d_{1}(t)+\frac{\partial f_{1}(\boldsymbol{x})}{\partial x_{2}} d_{2}(t)$.

Assumption $1 b(\boldsymbol{x})+\frac{\partial f_{1}(\boldsymbol{x})}{\partial x_{2}} b(\boldsymbol{x}) \neq 0$ for $\forall \boldsymbol{x} \in \mathbb{R}^{2}$.

Assumption $2|\dot{D}(t)|<L$ with $L$ as a known positive constant.

Assumption $3 \frac{\partial f_{1}(\boldsymbol{x})}{\partial x_{2}} f_{2}(\boldsymbol{x})+\frac{\partial f_{1}(\boldsymbol{x})}{\partial x_{1}}\left(x_{2}+f_{1}(\boldsymbol{x})\right)+$ $f_{2}(\boldsymbol{x})$ is continuous and bounded for the finite $\boldsymbol{x}$.

The control objective of this paper is to derive a control system for the system (1) such that: 1) $x_{1}$ converges to zero with the UBST tightly prescribed by one design parameter of the controller, in the presence of the mismatched disturbance $d_{1}(t)$ and matched disturbance $\left.d_{2}(t) .2\right)$ All signals in the closed-loop system remain bounded. 3) The control input $u$ is continuous.

Two definitions used throughout the paper are provided below. For a non-autonomous nonlinear system:

$\dot{\breve{\boldsymbol{x}}}=f(t, \breve{\boldsymbol{x}} ; \widehat{\boldsymbol{\varphi}}), \quad \breve{\boldsymbol{x}}\left(t_{0}\right)=\breve{\boldsymbol{x}}_{0}$

where $\breve{\boldsymbol{x}} \in \mathbb{R}^{n}$ is the system state vector, $\widehat{\varphi} \in \mathbb{R}^{w}$ denotes the vector of constant system parameters, and $f: \mathbb{R}_{>0} \times \mathbb{R}^{n} \rightarrow \mathbb{R}^{n}$ represents a nonlinear function such that $f(t, 0 ; \widehat{\boldsymbol{\varphi}})=0$, namely the origin $\breve{\boldsymbol{x}}=0$ is an equilibrium point of the system (3).

Definition 1 (Fixed-time stability, FxTS [14]) The origin of the system (3) exhibits FxTS if, for any initial conditions $\breve{\boldsymbol{x}}_{0}$, there exists a positive and bounded constant $\bar{T}$ such that for $\forall t \geq \bar{T}, \breve{\boldsymbol{x}}(t)=0$ holds.

Definition 2 (Prescribed-time stability, PTS [14]) The origin of the system (3) exhibits PTS if (i) It is of FxTS, (ii) For any $\bar{T}>0$, there exist some system parameters $\widehat{\boldsymbol{\varphi}}$ such that $\breve{\boldsymbol{x}}(t)=0$ holds for $\forall t \geq \bar{T}$.

\section{Main results}

It is well established that, by transforming the system into a perturbed integrator-like system, the typical active disturbance rejection control (ADRC) framework [36] is competent to handle mismatched disturbances. Hence, the control system is developed within 
the framework of ADRC, consisting of a PTESO for prescribed-time observation and a CPTSMC for prescribed-time stabilization. The UBST of observation errors and $x_{1}$ are each prescribed by one design parameter tightly. In addition, for practical applications, parameter settings that guarantee the boundedness of the control input and system signals are given, which was not explored in $[27,28]$.

\subsection{Prescribed-time ESO design}

The ESO in this section serves to reconstruct the unmeasurable $\dot{x}_{1}$ and $D(t)$. First, based on Eq. (2), we rewrite the system (1) as a third-order system:

$\dot{x}_{1}=\bar{x}_{2}, \quad \dot{\bar{x}}_{2}=H(\boldsymbol{x}, u)+\bar{x}_{3}, \quad \dot{\bar{x}}_{3}=\varepsilon(t)$

where $H(\boldsymbol{x}, u)=f_{2}(\boldsymbol{x})+\frac{\partial f_{1}(\boldsymbol{x})}{\partial x_{1}}\left(x_{2}+f_{1}(\boldsymbol{x})\right)+$ $\frac{\partial f_{1}(\boldsymbol{x})}{\partial x_{2}} f_{2}(\boldsymbol{x})+\left(b(\boldsymbol{x})+\frac{\partial f_{1}(\boldsymbol{x})}{\partial x_{2}} b(\boldsymbol{x})\right) u \cdot \varepsilon(t)=[-L, L]$ is a differential inclusion with $L$ in Assumption 2 .

Accordingly, by defining $\hat{x}_{1}, \hat{x}_{2}$, and $\hat{x}_{3}$ as the observed values of $x_{1}, \bar{x}_{2}$, and $\bar{x}_{3}$, respectively, an ESO with the switch logic is presented in the following form:

$$
\left\{\begin{array}{l}
\dot{\hat{x}}_{1}=\hat{x}_{2}+\varpi(t, T) \hbar_{1}\left(x_{1}-\hat{x}_{1}, T\right) \\
+(1-\varpi(t, T)) \vartheta_{1}\left(x_{1}-\hat{x}_{1}\right) \\
\dot{\hat{x}}_{2}=H(\boldsymbol{x}, u)+\hat{x}_{3}+\varpi(t, T) \hbar_{2}\left(x_{1}-\hat{x}_{1}, T\right) \\
\quad+(1-\varpi(t, T)) \vartheta_{2}\left(x_{1}-\hat{x}_{1}\right) \\
\dot{\hat{x}}_{3}=\varpi(t, T) \hbar_{3}\left(x_{1}-\hat{x}_{1}, T\right) \\
+(1-\varpi(t, T)) \vartheta_{3}\left(x_{1}-\hat{x}_{1}\right)
\end{array}\right.
$$

where $T$ equals the value of the desired settling time of the observation errors. $\varpi(t, T)$ is a switch function where $\varpi(t, T)=1$ for $t<t_{0}+T$ and $\varpi(t, T)=0$ for $t \geq t_{0}+T . \hbar_{i}\left(x_{1}-\hat{x}_{1}, T\right)$ and $\vartheta_{i}\left(x_{1}-\hat{x}_{1}\right)$ are correction terms that govern within the time intervals $\left[t_{0}, t_{0}+T\right)$ and $\left[t_{0}+T, \infty\right)$, respectively.

With $\tilde{x}_{1}=x_{1}-\hat{x}_{1}, \tilde{x}_{2}=\bar{x}_{2}-\hat{x}_{2}$ and $\tilde{x}_{3}=\bar{x}_{3}-\hat{x}_{3}$, the error model of the ESO (5) is depicted by:

$$
\left\{\begin{array}{l}
\dot{\tilde{x}}_{1}=\tilde{x}_{2}-\varpi(t, T) \hbar_{1}\left(\tilde{x}_{1}, T\right)-(1-\varpi(t, T)) \vartheta_{1}\left(\tilde{x}_{1}\right) \\
\dot{\tilde{x}}_{2}=\tilde{x}_{3}-\varpi(t, T) \hbar_{2}\left(\tilde{x}_{1}, T\right)-(1-\varpi(t, T)) \vartheta_{2}\left(\tilde{x}_{1}\right)(6) \\
\dot{\tilde{x}}_{3}=\varepsilon(t)-\varpi(t, T) \hbar_{3}\left(\tilde{x}_{1}, T\right)-(1-\varpi(t, T)) \vartheta_{3}\left(\tilde{x}_{1}\right)
\end{array}\right.
$$

In the following section, the design strategy of the ESO is illustrated in Subsection 3.1.1. Subsection 3.1.2 presents the design results. Finally, the PTS of observation errors $\left(\tilde{x}_{1}, \tilde{x}_{2}, \tilde{x}_{3}\right)$ with UBST $T$ is analysed in Subsection 3.1.3.

\subsubsection{Design strategy}

To ease comprehension, the design strategy is presented in advance. Inspired by the prescribed-time state observer [45], a monotone increasing function over time
$\mu_{1}=\frac{T}{T+t_{0}-t}$ is employed as the design basis. Obviously, $\mu_{1} \rightarrow \infty$ as $t \rightarrow t_{0}+T$. The design strategy within $\left[t_{0}, t_{0}+T\right)$ and $\left[t_{0}+T, \infty\right)$ is detailed as follows.

Within the time interval $\left[t_{0}, t_{0}+T\right)$, based on geometric transformations using the function $\mu_{1}$ (illustrated in Subsection 3.1.3), the observation error model (6) can be transformed into a third-order perturbed system given by:

$\left\{\begin{array}{l}\dot{\phi}_{1}=\phi_{2}-\iota_{1} \phi_{1} \\ \dot{\phi}_{2}=\phi_{3}-\iota_{2} \phi_{1} \\ \dot{\phi}_{3}=-\iota_{3} \phi_{1}+g(t, \varepsilon(t))\end{array}\right.$

where $\phi_{i}, i=1,2,3$ are the transformed states corresponding to $\tilde{x}_{i}, i=1,2,3$, respectively. $\iota_{i}, i=1,2,3$ are design parameters. $g(t, \varepsilon(t))$ denotes the mapped version of $\varepsilon(t)$, which may go to infinity as $t \rightarrow t_{0}+T$ due to the function $\mu_{1}$.

There exists a difference between our design strategy and that of the prescribed-time state observer in [45]. The strategy in [45] demands that $\phi_{i}, i=1,2,3$ remain bounded within the time interval $\left[t_{0}, t_{0}+T\right)$, which is definitely invalid for model (7) considering $g(t, \varepsilon(t))$. Consequently, the state observer [45] cannot be directly applied to observing time-varying disturbance. In this paper, we try to acquire the upper bounds of $\phi_{i}, i=1,2,3$ based on the detailed form of Eq. (7). Then, through the inverse transformation, the upper bounds of $\tilde{x}_{i}, i=1,2,3$ are obtained in the representation containing $\mu_{1}^{-1}$. Recalling that $\mu_{1} \rightarrow \infty$ as $t \rightarrow t_{0}+T$, we have $\mu_{1}^{-1} \rightarrow 0$ simultaneously. Therefore, even when $\phi_{i} \rightarrow \infty$, making $\tilde{x}_{i} \rightarrow 0$ as $t \rightarrow t_{0}+T$ is still probable.

Within the time interval $\left[t_{0}+T, \infty\right)$, the ESO switches to the finite-time ESO [47]. Due to $\tilde{x}_{i}\left(t_{0}+T\right)=$ 0 , observation errors remain unchanged thereafter, i.e., $\tilde{x}_{i}=0$ holds for $t \in\left[t_{0}+T, \infty\right)$.

\subsubsection{Design results}

Within the time interval $\left[t_{0}, t_{0}+T\right)$, the correction terms are contrived as:

$$
\begin{aligned}
& \hbar_{1}\left(\tilde{x}_{1}, T\right)=\left(\iota_{1}+3 \frac{3+\nu}{T} \mu_{1}\right) \tilde{x}_{1} \\
& \hbar_{2}\left(\tilde{x}_{1}, T\right)=\left(\iota_{2}-3 \frac{(3+\nu)(4+\nu)}{T^{2}} \mu_{1}^{2}\right) \tilde{x}_{1} \\
& +2 \frac{(3+\nu)}{T} \mu_{1} \hbar_{1}\left(\tilde{x}_{1}, T\right) \\
& \hbar_{3}\left(\tilde{x}_{1}, T\right)=\left(\iota_{3}+\frac{(3+\nu)(4+\nu)(5+\nu)}{T^{3}} \mu_{1}^{3}\right) \tilde{x}_{1} \\
& +\frac{(3+\nu)}{T} \mu_{1} \hbar_{2}\left(\tilde{x}_{1}, T\right)-\frac{(3+\nu)(4+\nu)}{T^{2}} \mu_{1}^{2} \hbar_{1}\left(\tilde{x}_{1}, T\right)
\end{aligned}
$$

where $\left(\iota_{1}, \iota_{2}, \iota_{3}\right)$ and $\nu$ are design parameters. $T>0$ prescribes the UBST. 
Within the time interval $\left[t_{0}+T, \infty\right)$, the correction terms are given by:

$\vartheta_{1}\left(\tilde{x}_{1}\right)=k_{1} L^{1 / 3}\left\lceil\tilde{x}_{1}\right\rfloor^{2 / 3}, \vartheta_{2}\left(\tilde{x}_{1}\right)=k_{2} L^{2 / 3}\left\lceil\tilde{x}_{1}\right\rfloor^{1 / 3}$

$\vartheta_{3}\left(\tilde{x}_{1}\right)=k_{3} L\left\lceil\tilde{x}_{1}\right\rfloor^{0}$

where $\left(k_{1}, k_{2}, k_{3}\right)$ are positive constants. Following [47], $k_{3}>1, k_{1}=3.34 k_{3}^{1 / 3}, k_{2}=5.3 k_{3}^{2 / 3}$.

Although multiple design parameters exist in the proposed ESO, the UBST is only prescribed by $T$. Other parameters can be tuned to acquire high transient performance, such as low peak values. The tuning suggestions are provided in Subsection 3.1.3.

\subsubsection{Prescribed-time stability analysis}

The observation error model within $\left[t_{0}, t_{0}+T\right)$ is:

$$
\left\{\begin{array}{l}
\dot{\tilde{x}}_{1}=\tilde{x}_{2}-\hbar_{1}\left(\tilde{x}_{1}, T\right) \\
\dot{\tilde{x}}_{2}=\tilde{x}_{3}-\hbar_{2}\left(\tilde{x}_{1}, T\right) \\
\dot{\tilde{x}}_{3}=\varepsilon(t)-\hbar_{3}\left(\tilde{x}_{1}, T\right)
\end{array}\right.
$$

Before proceeding to the PTS analysis of the ESO, two important time-varying geometric transformations are introduced in advance. Considering the transformations $\tilde{x}_{i} \mapsto \varsigma_{i}$ and $\varsigma_{i} \mapsto \phi_{i}$ defined by:

$\varsigma_{i}=\mu \tilde{x}_{i}, \quad \phi_{i}=\sum_{j=1}^{i} \rho_{i, j}^{*} \varsigma_{j}, i=1,2,3$

where $\mu=\mu_{1}^{3+\nu}, \rho_{i, j}^{*}=\bar{\rho}_{i, j} \mu_{1}^{i-j}, 1 \leq\{i, j\} \leq 3$, and:

$\bar{\rho}_{1,1}=1, \quad \bar{\rho}_{1,2}=0, \quad \bar{\rho}_{1,3}=0, \quad \bar{\rho}_{2,1}=-2 \frac{3+\nu}{T}$

$\bar{\rho}_{2,2}=1, \quad \bar{\rho}_{2,3}=0, \quad \bar{\rho}_{3,1}=\frac{(3+\nu)(4+\nu)}{T^{2}}$

$\bar{\rho}_{3,2}=-\frac{3+\nu}{T}, \quad \bar{\rho}_{3,3}=1$

Accordingly, the time-derivatives of $\varsigma_{i}$ and $\phi_{i}$ are given by:

$\dot{\varsigma}_{i}=\mu \dot{\tilde{x}}_{i}+\frac{(3+\nu) T^{3+\nu}}{\left(T+t_{0}-t\right)^{4+\nu}} \tilde{x}_{i}$
$\dot{\phi}_{i}=\sum_{j=1}^{i}\left(\bar{\rho}_{i, j} \mu_{1}^{i-j} \dot{\varsigma}_{j}+(i-j) \bar{\rho}_{i, j} \mu_{1}^{i-j-1} \frac{\mu_{1}^{2}}{T} \varsigma_{j}\right)$

Using Eqs. (13) and (15), the time-derivative of $\phi_{i}$ within the time interval $\left[t_{0}, t_{0}+T\right)$ is rewritten as:

$\dot{\phi}_{i}=\sum_{j=1}^{i}\left(\begin{array}{l}\bar{\rho}_{i, j} \mu_{1}^{i-j}\left(\mu \dot{\tilde{x}}_{j}+\frac{(3+\nu) T^{3+\nu}}{\left(T+t_{0}-t\right)^{4+\nu}} \tilde{x}_{j}\right. \\ +(i-j) \bar{\rho}_{i, j} \mu_{1}^{i-j-1} \frac{\mu_{1}^{2}}{T} \mu \tilde{x}_{j}\end{array}\right)$

Substitute Eq. (12) into Eq. (16). Through basic algebraic calculation, a perturbed system is obtained:

$$
\left\{\begin{array}{l}
\dot{\phi}_{1}=\phi_{2}-\iota_{1} \phi_{1} \\
\dot{\phi}_{2}=\phi_{3}-\iota_{2} \phi_{1} \\
\dot{\phi}_{3}=-\iota_{3} \phi_{1}+\mu \times \varepsilon(t)
\end{array}\right.
$$

The theorem about the PTS of $\tilde{x}_{i}$ in the presence of $\mu \times \varepsilon(t)$ is given below.
Theorem 1 Let the constants $\iota_{i}, i=1,2,3$ make the polynomial $s^{3}+\iota_{1} s^{2}+\iota_{2} s+\iota_{3}$ Hurwitz, $T>0, \nu>0$. Observation errors $\tilde{x}_{i}, i=1,2,3$ converge to the origin within the time interval $\left[t_{0}, t_{0}+T\right)$ and remain unchanged thereafter. Furthermore, the correction terms $\hbar_{i}\left(\tilde{x}_{1}, T\right), i=1,2,3$ remain bounded within the time interval $\left[t_{0}, t_{0}+T\right)$.

Proof The proof is divided into three steps. Step 1 briefly shows the PTS of $\tilde{x}_{i}$ in the presence of timeinvariant disturbance (i.e., $\varepsilon(t) \equiv 0$ ). In Step 2, the condition under time-varying disturbance within the time interval $\left[t_{0}, t_{0}+T\right)$ is discussed. Then, the convergence property of observation errors within the time interval $\left[t_{0}+T, \infty\right)$ is considered in Step 3 .

Step 1: If $\varepsilon(t) \equiv 0$, Eq. (17) degrades to:

$\left\{\begin{array}{l}\dot{\phi}_{1}=\phi_{2}-\iota_{1} \phi_{1} \\ \dot{\phi}_{2}=\phi_{3}-\iota_{2} \phi_{1} \\ \dot{\phi}_{3}=-\iota_{3} \phi_{1}\end{array}\right.$

Considering the Hurwitz polynomial $s^{3}+\iota_{1} s^{2}+\iota_{2} s+$ $\iota_{3}, \phi_{i, i=1,2,3}$ remain bounded within the time interval $\left[t_{0}, t_{0}+T\right)$. By reversing the transformations in Eq. (13), there is:

$$
\begin{aligned}
& \tilde{x}_{i}=\mu^{-1} \varsigma_{i}, \quad \varsigma_{1}=\phi_{1} \\
& \varsigma_{2}=\phi_{2}-\bar{\rho}_{2,1} \mu_{1} \phi_{1} \\
& \varsigma_{3}=\phi_{3}-\bar{\rho}_{3,1} \mu_{1}^{2} \phi_{1}-\bar{\rho}_{3,2} \mu_{1}\left(\phi_{2}-\bar{\rho}_{2,1} \mu_{1} \phi_{1}\right)
\end{aligned}
$$

Since $\mu=\mu_{1}^{3+\nu}$ with $\nu>0$, and $\mu_{1} \rightarrow \infty$ as $t \rightarrow$ $t_{0}+T$, it is straightforward to obtain that $\left|\tilde{x}_{i}\right| \rightarrow 0$ as $t \rightarrow t_{0}+T$, i.e., PTS with the prescribed UBST $T$ is realized. Additionally, according to Eqs. (8)-(10) and $(19), \hbar_{i}\left(\tilde{x}_{1}, T\right)$ remains bounded within the time interval $\left[t_{0}, t_{0}+T\right)$ and converges to zero as $t \rightarrow t_{0}+T$.

Step 2: If $\varepsilon(t)$ is not uniformly equal to zero, the time trajectories of $\phi_{i, i=1,2,3}$ in Eq. (17) are:

$$
\begin{aligned}
& \phi(t)=\underbrace{e^{\boldsymbol{A}_{\phi}\left(t-t_{0}\right)} \boldsymbol{\phi}\left(t_{0}\right)}_{\text {Part1 }}+\underbrace{\int_{t_{0}}^{t} e^{\boldsymbol{A}_{\boldsymbol{\phi}}(t-\gamma)} \boldsymbol{B}_{\phi} \mu \varepsilon(\gamma) \mathrm{d} \gamma}_{\text {part2 }} \\
& \Rightarrow|\boldsymbol{\phi}(t)| \leq\left|e^{\boldsymbol{A}_{\phi}\left(t-t_{0}\right)} \boldsymbol{\phi}\left(t_{0}\right)\right|+\left|\int_{t_{0}}^{t} e^{\boldsymbol{A}_{\phi}(t-\gamma)} \boldsymbol{B}_{\phi} \mu \varepsilon(\gamma) \mathrm{d} \gamma\right|
\end{aligned}
$$

where $\boldsymbol{\phi}=\left[\begin{array}{l}\phi_{1} \\ \phi_{2} \\ \phi_{3}\end{array}\right], \boldsymbol{A}_{\boldsymbol{\phi}}=\left[\begin{array}{lll}-\iota_{1} & 1 & 0 \\ -\iota_{2} & 0 & 1 \\ -\iota_{3} & 0 & 0\end{array}\right]$, and $\boldsymbol{B}_{\boldsymbol{\phi}}=\left[\begin{array}{l}0 \\ 0 \\ 1\end{array}\right]$.

With the use of Eq. (19), we have:

$$
\begin{aligned}
& \left|\tilde{x}_{1}\right|=\mu^{-1}\left|\varsigma_{1}\right|=\mu^{-1}\left|\phi_{1}\right| \\
& \left|\tilde{x}_{2}\right|=\mu^{-1}\left|\varsigma_{2}\right| \leq \mu^{-1}\left|\phi_{2}\right|+\mu^{-1} \bar{\rho}_{2,1} \mu_{1}\left|\phi_{1}\right| \\
& \left|\tilde{x}_{3}\right|=\mu^{-1}\left|\varsigma_{3}\right| \leq \mu^{-1}\left(\begin{array}{l}
\left|\phi_{3}\right|+\bar{\rho}_{3,2} \mu_{1}\left|\phi_{2}\right| \\
+\left(\bar{\rho}_{3,1}+\bar{\rho}_{3,2} \bar{\rho}_{2,1}\right) \mu_{1}^{2}\left|\phi_{1}\right|
\end{array}\right)
\end{aligned}
$$


Therefore, although $\phi$ may escape to infinity driven by $\mu \varepsilon(t),\left(\left|\tilde{x}_{1}\right|,\left|\tilde{x}_{2}\right|,\left|\tilde{x}_{3}\right|\right)$ may still approach zero as $t \rightarrow$ $t_{0}+T$ due to $\mu^{-1}$. As shown in Eq. (20), the value of $\phi_{i}$ comprises two parts, in which 'Part1' denotes the zeroinput response of the linear unperturbed system (18), and 'Part2' stands for the effects of $\mu \varepsilon(t)$. Substituting Eq. (20) into Eq. (21) leads to:

$\left.\begin{array}{rl}\left|\tilde{x}_{1}\right| \leq & \left.\begin{array}{l}\underbrace{\text { term } 1}_{\mu^{-1}\left|e^{\boldsymbol{A}_{\phi}\left(t-t_{0}\right)} \boldsymbol{\phi}\left(t_{0}\right)\right|_{1}} \\ +\mu^{-1}\left|\int_{t_{0}}^{t} e^{\boldsymbol{A}_{\phi}(t-\gamma)} \boldsymbol{B}_{\phi} \mu \varepsilon(\gamma) \mathrm{d} \gamma\right|_{1}\end{array}\right) \\ \left|\tilde{x}_{2}\right| \leq\left\{\begin{array}{l}\underbrace{\mu^{-1}\left(\begin{array}{l}\left|e^{\boldsymbol{A}_{\phi}\left(t-t_{0}\right)} \boldsymbol{\phi}\left(t_{0}\right)\right|_{2} \\ +\bar{\rho}_{2,1} \mu_{1}\left|e^{\boldsymbol{A}_{\phi}\left(t-t_{0}\right)} \boldsymbol{\phi}\left(t_{0}\right)\right|_{1}\end{array}\right)}_{\text {term2 }} \\ +\mu^{-1}\left|\int_{t_{0}}^{t} e^{\boldsymbol{A}_{\phi}(t-\gamma)} \boldsymbol{B}_{\phi} \mu \varepsilon(\gamma) \mathrm{d} \gamma\right|_{2} \\ +\mu^{-1} \bar{\rho}_{2,1} \mu_{1}\left|\int_{t_{0}}^{t} e^{\boldsymbol{A}_{\phi}(t-\gamma)} \boldsymbol{B}_{\phi} \mu \varepsilon(\gamma) \mathrm{d} \gamma\right|_{1}\end{array}\right.\end{array}\right\}$

$\left|\tilde{x}_{3}\right| \leq\left\{\begin{array}{l}\underbrace{\left(\begin{array}{l}\mu^{-1} Y \mu_{1}^{2}\left|e^{\boldsymbol{A}_{\phi}\left(t-t_{0}\right)} \boldsymbol{\phi}\left(t_{0}\right)\right|_{1} \\ +\mu^{-1} \bar{\rho}_{3,2} \mu_{1}\left|e^{\boldsymbol{A}_{\phi}\left(t-t_{0}\right)} \boldsymbol{\phi}\left(t_{0}\right)\right|_{2} \\ +\mu^{-1}\left|e^{\boldsymbol{A}_{\phi}\left(t-t_{0}\right)} \boldsymbol{\phi}\left(t_{0}\right)\right|_{3}\end{array}\right)}_{\text {term } 3} \\ +\left(\begin{array}{l}\mu^{-1} Y \mu_{1}^{2}\left|\int_{t_{0}}^{t} e^{\boldsymbol{A}_{\phi}(t-\gamma)} \boldsymbol{B}_{\phi} \mu \varepsilon(\gamma) \mathrm{d} \gamma\right|_{1} \\ +\mu^{-1} \bar{\rho}_{3,2} \mu_{1}\left|\int_{t_{0}}^{t} e^{\boldsymbol{A}_{\phi}(t-\gamma)} \boldsymbol{B}_{\phi} \mu \varepsilon(\gamma) \mathrm{d} \gamma\right|_{2} \\ +\mu^{-1}\left|\int_{t_{0}}^{t} e^{\boldsymbol{A}_{\phi}(t-\gamma)} \boldsymbol{B}_{\phi} \mu \varepsilon(\gamma) \mathrm{d} \gamma\right|_{3}\end{array}\right.\end{array}\right\}$

where $Y=\bar{\rho}_{3,1}+\bar{\rho}_{3,2} \bar{\rho}_{2,1}$, and the subscript ' $i$ ' outside the absolute value symbol marks the $i_{t h}$ element of the corresponding vectors.

As analysed in Step 1 and regarding $\nu>0$, 'term 1', 'term 2', and 'term 3' in Eqs. (22)-(24) tend to be zero as $t \rightarrow t_{0}+T$. Thus, the effects of $\mu \varepsilon(t)$ determine whether the PTS of observation errors in the presence of time-varying disturbances can be achieved.

Given the structure of the system $(17), \mu \varepsilon(t)$ impacts the system states most violently if $|\varepsilon(t)| \equiv L$ within the time interval $\left[t_{0}, t_{0}+T\right)$. In this case, caused by $\mu \varepsilon(t) \rightarrow \infty$ as $t \rightarrow t_{0}+T,\left(\phi_{1}, \phi_{2}, \phi_{3}\right)$ tends to have the same sign as $\varepsilon(t)$ does. One shall define the interval $N:=\left[t_{0}, t_{0}+T\right), \bar{\phi}:=\sup _{\Delta, t \in N}\left|\phi_{1}(t)\right|$, where $\Delta$ denotes the criterion that either $\phi_{1}(t) \varepsilon(t)<0$, $\phi_{1}(t) \phi_{3}(t)<0$ or $\phi_{1}(t) \phi_{2}(t)<0$ is valid (if this criterion is not met within the time interval $N$, then $\bar{\phi}=0$ ). Given that the polynomial $s^{3}+\iota_{1} s^{2}+\iota_{2} s+\iota_{3}$ is Hurwitz and $\left(\phi_{1}\left(t_{0}\right), \phi_{2}\left(t_{0}\right), \phi_{3}\left(t_{0}\right)\right)$ are finite numbers, it can be inferred that $\bar{\phi}$ is a finite number. Then, based on Eq. (17), the upper bounds of $\left(\left|\phi_{1}\right|,\left|\phi_{2}\right|,\left|\phi_{3}\right|\right)$ read:

$\left|\phi_{3}(t)\right| \leq\left(t-t_{0}\right) \bar{\phi} \iota_{3}+L \int_{t_{0}}^{t} \mu(\tau) \mathrm{d} \tau+\left|\phi_{3}\left(t_{0}\right)\right|$ $\leq\left(t-t_{0}\right) \bar{\phi} \iota_{3}+\left|\phi_{3}\left(t_{0}\right)\right|+\frac{L T^{\nu+3}}{\nu+2} \frac{T^{\nu+2}-\left(T+t_{0}-t\right)^{\nu+2}}{\left(T+t_{0}-t\right)^{\nu+2} T^{\nu+2}}$ $\left|\phi_{2}(t)\right| \leq \int_{t_{0}}^{t}\left|\phi_{3}(\tau)\right| \mathrm{d} \tau+\left(t-t_{0}\right) \bar{\phi} \iota_{2}+\left|\phi_{2}\left(t_{0}\right)\right|$

$\leq \frac{\left(t-t_{0}\right)^{2}}{2} \bar{\phi} \iota_{3}+L \frac{T^{\nu+3}}{\nu+2}\left(\begin{array}{c}\frac{1}{(\nu+1)\left(T+t_{0}-t\right)^{\nu+1}} \\ -\frac{1}{(\nu+1) T^{\nu+1}}-\frac{t-t_{0}}{T^{\nu+2}}\end{array}\right)$

$+\left(t-t_{0}\right) \bar{\phi} \iota_{2}+\left(t-t_{0}\right)\left|\phi_{3}\left(t_{0}\right)\right|+\left|\phi_{2}\left(t_{0}\right)\right|$

$\left|\phi_{1}(t)\right| \leq \int_{t_{0}}^{t}\left|\phi_{2}(\tau)\right| \mathrm{d} \tau+\left|\phi_{1}\left(t_{0}\right)\right|+\left(t-t_{0}\right) \bar{\phi} \iota_{1}$

$\leq \frac{\left(t-t_{0}\right)^{3}}{6} \bar{\phi} \iota_{3}+\frac{\left(t-t_{0}\right)^{2}\left|\phi_{3}\left(t_{0}\right)\right|}{2}+\left(t-t_{0}\right) \bar{\phi} \iota_{1}$

$+L \frac{T^{\nu+3}}{\nu+2}\left(\begin{array}{l}\frac{1}{\nu(\nu+1)\left(T+t_{0}-t\right)^{\nu}}-\frac{1}{\nu(\nu+1) T^{\nu}} \\ -\frac{t-t_{0}}{(\nu+1) T^{\nu+1}}-\frac{\left(t-t_{0}\right)^{2}}{2 T^{\nu+2}}\end{array}\right)$

$+\left(t-t_{0}\right)\left|\phi_{2}\left(t_{0}\right)\right|+\left|\phi_{1}\left(t_{0}\right)\right|+\frac{\left(t-t_{0}\right)^{2} \bar{\phi} \iota_{2}}{2}$

Subsequently, on the basis of Eq. (21), the upper bounds of $\left(\left|\tilde{x}_{1}\right|,\left|\tilde{x}_{2}\right|,\left|\tilde{x}_{3}\right|\right)$ are derived as:

$\left|\tilde{x}_{1}\right| \leq \frac{\left(t-t_{0}\right)^{3}}{6 \mu} \bar{\phi} \iota_{3}+\frac{\left(t-t_{0}\right) \bar{\phi} \iota_{1}}{\mu}+\frac{\left(t-t_{0}\right)^{2} \bar{\phi} l_{2}}{2 \mu}$
$+L \frac{T^{\nu+3}}{(\nu+2) \mu}\left(\begin{array}{l}\frac{1}{\nu(\nu+1)\left(T+t_{0}-t\right)^{\nu}}-\frac{1}{\nu(\nu+1) T^{\nu}} \\ -\frac{t-t_{0}}{(\nu+1) T^{\nu+1}}-\frac{\left(t-t_{0}\right)^{2}}{2 T^{\nu+2}}\end{array}\right)$
$+\frac{\left(t-t_{0}\right)^{2}\left|\phi_{3}\left(t_{0}\right)\right|}{2 \mu}+\frac{\left(t-t_{0}\right)\left|\phi_{2}\left(t_{0}\right)\right|}{\mu}+\frac{\left|\phi_{1}\left(t_{0}\right)\right|}{\mu}$

$\left|\tilde{x}_{2}\right| \leq \frac{\mu_{1} \bar{\rho}_{2,1}\left|\phi_{1}\right|}{\mu}+\frac{\left(t-t_{0}\right)^{2}}{2 \mu} \bar{\phi} \iota_{3}+\frac{\left(t-t_{0}\right)\left|\phi_{3}\left(t_{0}\right)\right|}{\mu}$

$+\frac{L T^{\nu+3}}{(\nu+2) \mu}\left(\begin{array}{c}\frac{1}{(\nu+1)\left(T+t_{0}-t\right)^{\nu+1}} \\ -\frac{1}{(\nu+1) T^{\nu+1}}-\frac{t-t_{0}}{T^{\nu+2}}\end{array}\right)$

$+\frac{\left(t-t_{0}\right) \bar{\phi} \iota_{2}}{\mu}+\frac{\left|\phi_{2}\left(t_{0}\right)\right|}{\mu}$

$\left|\tilde{x}_{3}\right| \leq \frac{\left(\bar{\rho}_{3,1}+\bar{\rho}_{3,2} \bar{\rho}_{2,1}\right) \mu_{1}^{2}\left|\phi_{1}\right|}{\mu}+\frac{\mu_{1} \bar{\rho}_{3,2}\left|\phi_{2}\right|}{\mu}+\frac{\left(t-t_{0}\right) \bar{\phi} \iota_{3}}{\mu}$

$+\frac{\left|\phi_{3}\left(t_{0}\right)\right|}{\mu}+\frac{L T^{\nu+3}}{(\nu+2) \mu}\left(\frac{1}{\left(T+t_{0}-t\right)^{\nu+2}}-\frac{1}{T^{\nu+2}}\right)$

which can be represented in the following general form:

$\left|\tilde{x}_{i}\right| \leq \frac{G_{i 1}}{\mu}+\frac{G_{i 2}}{\mu_{1}^{4-i}}, i=1,2,3$

where $G_{i 1}$ and $G_{i 2}$ are bounded numbers, of which the detailed form is easily accessible through Eqs. (28)-(30) and omitted here for brevity.

Recalling $\mu=\mu_{1}{ }^{3+\nu}$ and $\nu>0$, it is clear that the observation errors $\left(\tilde{x}_{1}, \tilde{x}_{2}, \tilde{x}_{3}\right)$ are driven to zero as $t \rightarrow$ $t_{0}+T$. Thus, the PTS of observation errors with UBST $T$ has been achieved. Note that the precise knowledge of $L$ is not required within the time interval $\left[t_{0}, t_{0}+\right.$ $T$ ). Once $L$ is bounded, the PTS of observation errors remains unchanged.

Furthermore, by using Eqs. (8)-(10) and (28)-(30), the correction terms $\hbar_{i}\left(\tilde{x}_{1}, T\right)$ follow:

$\lim _{t \rightarrow t_{0}+T}\left|\hbar_{1}\left(\tilde{x}_{1}, T\right)\right| \rightarrow 0$

$\lim _{t \rightarrow t_{0}+T}\left|\hbar_{2}\left(\tilde{x}_{1}, T\right)\right| \rightarrow 0$ 
$\left|\hbar_{3}\left(\tilde{x}_{1}, T\right)\right| \leq\left\{\begin{array}{l}\left(\iota_{3}+\frac{(3+\nu)(4+\nu)(5+\nu)}{T^{3}} \mu_{1}^{3}\right)\left|\tilde{x}_{1}\right| \\ +\frac{(3+\nu)}{T} \mu_{1}\left|\hbar_{2}\left(\tilde{x}_{1}, T\right)\right| \\ +\frac{(3+\nu)(4+\nu)}{T^{2}} \mu_{1}^{2}\left|\hbar_{1}\left(\tilde{x}_{1}, T\right)\right|\end{array}\right\}$

$\Rightarrow \lim _{t \rightarrow t_{0}+T}\left|\hbar_{3}\left(\tilde{x}_{1}, T\right)\right| \leq L \frac{(3+\nu)(4+\nu)(5+\nu)+6(3+\nu)^{2}(7+2 \nu)}{(\nu+2)(\nu+1) \nu}$

Here comes to a valid conclusion that the correction terms $\hbar_{i}\left(\tilde{x}_{1}, T\right)$ for $i=1,2,3$ remain bounded within the time interval $\left[t_{0}, t_{0}+T\right)$. As $t \rightarrow t_{0}+T, \hbar_{1}\left(\tilde{x}_{1}, T\right)$ and $\hbar_{2}\left(\tilde{x}_{1}, T\right)$ tend to be zero, while $\hbar_{3}\left(\tilde{x}_{3}, T\right)$ converges to a non-zero number. Hence, a weak oscillation of $\hat{x}_{3}$ may occur at the switch time $t=t_{0}+T$ in practical applications with finite sampling frequency, resulting in trivial influence on the observation performance.

Step 3: Within the time interval $\left[t_{0}+T, \infty\right)$, the ESO (5) changes into the finite-time ESO [47], and the observation error model is:

$\left\{\begin{array}{l}\dot{\tilde{x}}_{1}=\tilde{x}_{2}-k_{1} L^{1 / 3}\left\lceil\tilde{x}_{1}\right\rfloor^{2 / 3} \\ \dot{\tilde{x}}_{2}=\tilde{x}_{3}-k_{2} L^{2 / 3}\left\lceil\tilde{x}_{1}\right\rfloor^{1 / 3} \\ \dot{\tilde{x}}_{3}=\varepsilon(t)-k_{3} L\left\lceil\tilde{x}_{1}\right\rfloor^{0}\end{array}\right.$

Given that $\tilde{x}_{i}\left(t_{0}+T\right)=0$ holds, according to [47], $\tilde{x}_{i}\left(t_{0}+T\right), i=1,2,3$ are kept at the origin. Hereby, the proof of Theorem 1 is complete.

Remark 1 Although the form of the proposed ESO (5) is similar to the prescribed-time state observer [45], proof of the robustness against the time-varying disturbance is provided exclusively in this paper, resulting in a significantly extended application field. Moreover, recalling the design strategy in [45], $\phi_{i}(t)$ is required to be bounded within the time interval $\left[t_{0}, t_{0}+T\right)$, which is conservative to some extent. In this paper, we show that the PTS can be realized even when the state $\phi_{i}(t)$ becomes infinite, and accordingly, the selection range of $\nu$ is enlarged from $\nu \geqslant 1$ to $\nu>0$. In addition, due to $\varepsilon(t), \hbar_{3}\left(\tilde{x}_{3}, T\right)$ no longer approaches zero as $t \rightarrow t_{0}+T$. This is different from the conclusion in [45].

Remark 2 (Peaking value problem analysis) Due to the utilization of high gain correction terms, a high-gain linear ESO suffers a severe peaking value problem [37]. The nonlinear ESOs [38-41] employ fractional power functions to reduce the peaking values. Regarding the proposed $\operatorname{ESO}(5), \mu_{1}\left(t_{0}\right)=1$, and $\iota_{i, i=1,2,3}$ can be chosen to be sufficiently small without any influence on the UBST $T$. Therefore, for conditions with large initial observation errors, the correction terms $\hbar_{i}\left(\tilde{x}_{1}, T\right)$ have small initial values, and thus, the peaking value problem can be better addressed compared to the ESOs [38-41].

Remark 3 (Advantages over the PTESO [29]) To the authors' best knowledge, the only existing ESO with the UBST prescribed by one parameter is presented in [29]. However, this ESO [29] has complicated tuning procedures, in which two linear matrix inequalities need to be solved to obtain appropriate parameters. By contrast, for PTESO (5), it is quite easy to select $\left(\iota_{1}, \iota_{2}, \iota_{3}\right)$, which satisfies the Hurwitz condition. Moreover, when extending the order of the ESO to serve for higherorder systems, the parameter tuning complexity of the PTESO [29] increases rapidly considering the linear matrix inequalities. However, by using the geometric transformations [45] and deduction lines in this paper, a higher-order PTESO can be directly acquired by making $s^{n}+\iota_{1} s^{n-1}+\cdots+\iota_{n}$ Hurwitz, which is much more convenient. Most importantly, the gains of the PTESO in [29] grow much faster than those of the PTESO (5). Note that the PTESO in [29] utilizes the time-varying function $\sec ^{2}\left(\frac{\pi t}{2\left(T+t_{0}\right)}\right)$. With the use of L'Hopital's rule [48], there is $\lim _{t \rightarrow t_{0}+T} \sec ^{2}\left(\frac{\pi t}{2\left(T+t_{0}\right)}\right) / \mu_{1} \rightarrow \infty$. Considering the limited computation memory and finite sampling frequency of practical applications, PTESO (5) has a higher practical application value.

Remark 4 (Conservatism of the prescribed UBST) The estimated UBST of the finite-time or fixed-time ESOs [38-41] is conservative. By contrast, the newly proposed prescribed-time differentiators $[42,43]$ realized significantly reduced slack between the actual settling time and the estimated UBST. The proposed PTESO (5) further reduces the conservatism compared to the differentiators $[42,43]$. As shown in the proof, observation errors $\tilde{x}_{i}$ approach zero as $t \rightarrow t_{0}+T$. That is to say, conservatism of the prescribed UBST of the PTESO (5) is almost eliminated, resulting from the time-varying gain $\mu_{1}$ that grows to infinity as $t \rightarrow t_{0}+T$.

Remark 5 (Parameter tuning advice) The UBST of the proposed PTESO is determined by only one design parameter $T$ and trivially affected by other design parameters from the theoretical perspective. Therefore, once the parameters $\left(\iota_{i}, k_{i}, \nu\right)$ have been settled following Theorem 1, tuning $T$ is the sole remaining work for different observation temporal demands. However, in practical applications, the finite sampling frequency may cause the observation precision loss and the oscillation of $\hat{x}_{3}$ at the switch time $t=t_{0}+T$. As indicated in Eqs. (28)-(30) and (34), a larger $\nu$ can make $\left|\tilde{x}_{i}\right|$ reduce to small values at a faster rate and decrease the limit value of $\hbar_{3}\left(\tilde{x}_{3}, T\right)$ at $t=t_{0}+T$. Therefore, increasing $\nu$ is suggested when facing the previous problems.

Remark 6 As discussed in [29,42,43], unbounded gains may be problematic under noise, which is a common deficiency for the present prescribed-time observers. Implementing the switch slightly earlier than $t=t_{0}+T$ is a simple solution to overcome this problem at the trivial sacrifice of observation precision. Filtering $x_{1}$ by a 
first-order low-pass filter before utilizing the proposed ESO is another feasible choice. It is challenging to design a PTESO with bounded time-varying gains, and we will consider this in future work.

3.2 PTESO-based continuous prescribed-time sliding mode control

This section first presents a novel prescribed-time convergent form with higher transient performance than that of $[27,28]$. Then, a CPTSMC is derived based on the novel convergent form and the observed states from the PTESO. A rigorous stability proof considering the transient period of the PTESO is provided without using the 'separation principle' [25]. Ratio restrictions on the convergence rate of the ESO and the controller are thus relieved. Furthermore, the boundedness of the control input and system signals is proved, which is the key concern for practical applications.

\subsubsection{Novel prescribed-time convergent form design}

Considering the system (3), for a positive-definite function $V(t, \breve{\boldsymbol{x}})$ with $V(t, 0) \equiv 0$, a prescribed-time convergent form $\dot{V}(t, \breve{\boldsymbol{x}})=\frac{-\left(e^{V(t, \breve{\boldsymbol{x}})}-1\right) \eta}{e^{V(t, \breve{\boldsymbol{x}})}\left(t_{0}+t_{f}-t\right)}$ was proposed in $[27,28]$, which achieves $V\left(t_{0}+t_{f}\right)=0$ and $\dot{V}\left(t_{0}+t_{f}\right)=0$ in the continuous-time sense. Thus, if the system driven by the controller follows the foregoing convergent form, UBST of the system can be prescribed by a single design parameter $t_{f}$.

However, as shown from the simulation results in [27,28], $V$ satisfying the convergent form hits the origin aggressively (i.e., $\dot{V}$ is sizeable when $V$ is near zero). As a consequence, when applying methods $[27,28]$ to practical applications with finite sampling frequency, severe precision loss and intolerably large control input occur with a high probability as the time approaches the prescribed UBST. This fact motivates us to employ an additional power term $a$ to derive a novel prescribedtime convergent form, which realizes a mild arrival at the origin at the trivial sacrifice of computational complexity. The theorem for the new convergent form is given as follows.

Theorem 2 Let $\mathbb{D} \subset \mathbb{R}^{n}$ be a domain containing the point $\breve{\boldsymbol{x}}=0$ of system (3). Let $h_{1}(\breve{\boldsymbol{x}})$ and $h_{2}(\breve{\boldsymbol{x}})$ be two continuous positive definite functions in $\mathbb{D}$. If there exist two positive real numbers $\eta>1$ and $a \leq 1$, and a real-valued continuously differentiable function $V$ : $I \times \mathbb{D} \rightarrow \mathbb{R}_{\geq 0}$, where $I$ denotes a finite time interval and $I=\left[t_{0}, t_{0}+t_{f}\right)$, such that:

i) $h_{1}(\breve{\boldsymbol{x}}) \leq V(t, \breve{\boldsymbol{x}}) \leq h_{2}(\breve{\boldsymbol{x}}), \forall t \in I, \forall \breve{\boldsymbol{x}} \in \mathbb{D} \backslash\{0\}$.

ii) $V(t, 0) \equiv 0, \forall t \in I$. iii) $\dot{V} \leq \frac{\eta\left(e^{V^{a}}-1\right)}{-a V^{a-1} e^{V^{a}}\left(t_{f}+t_{0}-t\right)}, \forall V \neq 0, \forall t \in I$.

then $\breve{\boldsymbol{x}}=0$ exhibits PTS with the prescribed UBST $t_{f}$. Moreover, if

$\dot{V}=\frac{\eta\left(e^{V^{a}}-1\right)}{-a V^{a-1} e^{V^{a}}\left(t_{f}+t_{0}-t\right)}, \forall V \neq 0, \forall t \in I$

then $t=t_{0}+t_{f}$ is the actual settling time for $\breve{\boldsymbol{x}}$ (i.e., $\breve{\boldsymbol{x}} \rightarrow 0$ as $t \rightarrow t_{0}+t_{f}$ ).

Proof Please see Appendix A.

Remark 7 (Advantage over the convergent form [1418]) The remarkable advantage of the proposed Eq. (36) over the convergent form in [14-18] (for example, $\dot{V}(\breve{\boldsymbol{x}})=-\frac{1}{(1-\bar{p}) t_{f}} \frac{\kappa(V(\breve{\boldsymbol{x}}))^{\bar{p}}}{\kappa^{\prime}(V(\breve{\boldsymbol{x}}))}, \forall \breve{\boldsymbol{x}} \in \mathbb{R}^{n} \backslash\{0\}$ in [14] with $t_{f}$ as the prescribed UBST) is its substantially less conservativeness in meeting the temporal demand. For the convergent form in [14], $t=t_{0}+t_{f}$ becomes the actual settling time only when the norm of $\breve{\boldsymbol{x}}\left(t_{0}\right)$ is infinite, and the actual settling time with small initial values is much smaller than the prescribed UBST, which may cause excessive control efforts. By contrast, $V$ satisfying Eq. (36) converges to zero precisely at $t=t_{0}+t_{f}$ regardless of initial values. Excessive control efforts under small initial values are avoided.

Remark 8 (Advantage over the convergent form [29])The convergent form [29] also realizes the precise arrival at the origin at $t=t_{0}+t_{f}$ regardless of the initial values. However, similar to Remark 3, considering the time-varying gain $\sec ^{2}\left(\frac{\pi t}{2\left(t_{f}+t_{0}\right)}\right)$ [29], it is easy to obtain $\lim _{t \rightarrow t_{0}+t_{f}} \sec ^{2}\left(\frac{\pi t}{2\left(t_{f}+t_{0}\right)}\right) / \frac{1}{t_{f}+t_{0}-t} \rightarrow \infty$ with L'Hopital's rule [48]. Thus, the time-varying gain [29] grows much faster than the gain in Eq. (36). Regarding the limited computation memory and finite sampling frequency of practical applications, the convergent form (36) has higher practical application values.

The following property reveals the internal mechanism of $a$ in adjusting the transient process, together with the tuning rule for the higher transient performance.

Property 1 Assume $\chi(t)$ as a solution to Eq. (36). With the same $t_{f}, \eta$ and initial value $\chi\left(t_{0}\right)$, there always exists a time interval $\left[t_{0}+t_{f}-m, t_{0}+t_{f}\right)$ with $m>0$, such that for $t \in\left[t_{0}+t_{f}-m, t_{0}+t_{f}\right),|\dot{\chi}(t)|$ decreases along with decreasing $a$.

Proof Please see Appendix B.

Therefore, by reducing the value of $a$, the solution to Eq. (36) can reach zero more mildly. To make the 


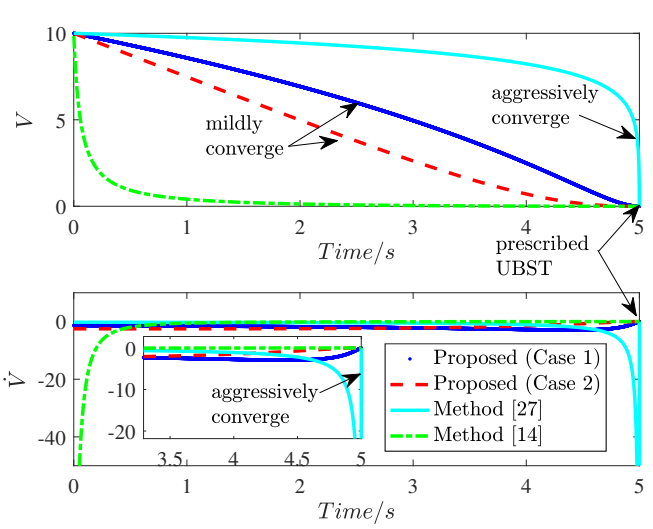

Fig. 1 Curves of $V$ and $\dot{V}$ in the continuous-time sense

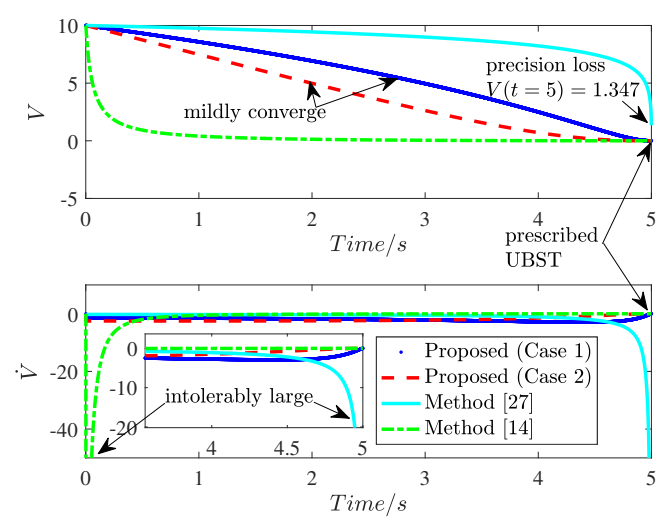

Fig. 2 Curves of $V$ and $\dot{V}$ under simulation step size 1e-3 s

analysis results more intuitive, the prescribed-time convergent differential equations in $[14,27]$ are adopted in Example 1 as the comparison to the proposed convergent form (36).

Example 1 In the simulation, $V\left(t_{0}\right)=10, t_{0}=0 \mathrm{~s}$, other parameter settings are presented in Table 1. Two cases of the proposed form are included to verify the effect of $a$. Time histories of $(V, \dot{V})$ in the continuoustime sense and those under simulation step size 1e-3 s are shown in Figs. 1 and 2, respectively.

As shown in Figs. 1 and 2, although the prescribed UBST is $t=5 \mathrm{~s}$ in all simulation conditions, the actual settling time for the method [14] is shorter than $2 \mathrm{~s}$, resulting in the maximum value of $|\dot{V}|$ larger than 50 in Figs. 1 and 2. By contrast, regarding the proposed method, the maximum $|\dot{V}|$ of cases 1 and 2 in Fig. 1 equal 2.51 and 2.918, respectively. In Fig. 2, the corresponding values are 2.51 and 2.927 . Therefore, the probability of the control input saturation can be significantly reduced without violating the time constraints when the proposed convergent form is applied. Moreover, due to the aggressive convergence of the method
[27], severe precision loss arises in the simulation with step size 1e-3 s. In comparison, precise convergence is still realized by the proposed method. Even worse, the imprecise convergence of the method [27] makes $\dot{V}$ intolerably large, which essentially represents the excessive control efforts. While, $\dot{V}$ converges to zero in the simulation of the proposed method, indicating the higher practical application value of Eq. (36) compared to the method [27].

The efficiency of $a$ in adjusting the transient performance is also validated. For case 2 with a smaller $a$, $\dot{V}$ is smaller when $V$ is near zero, which is in line with Property 1. Thus, when oscillation or large overshoot occurs in practical applications, lowering the value of $a$ would be helpful.

\subsubsection{CPTSMC design}

The CPTSMC derived for the system (1) is in the form of the integral SMC. The structure of the integral sliding surface $S$ and $u(t)$ is given in advance:

$$
\begin{aligned}
& S=\hat{x}_{2}-\int_{t_{0}}^{t}\left(b(\boldsymbol{x})+\frac{\partial f_{1}(\boldsymbol{x})}{\partial x_{2}} b(\boldsymbol{x})\right) u_{\text {norm }}(\tau) \mathrm{d} \tau \\
& u(t)=u_{\text {norm }}(t)+u_{\text {ast }}(t)
\end{aligned}
$$

where $\hat{x}_{2}$ denotes the observation value of $\dot{x}_{1}$ provided by the PTESO. The control components $u_{\text {norm }}$ and $u_{\text {ast }}$ serve to guarantee the prescribed-time convergence towards the equilibrium point and prevent the system from leaving the sliding surface, respectively. By setting $\hat{x}_{2}\left(t_{0}\right)$ to zero, $S\left(t_{0}\right)=0$ holds.

By using Eqs. (1), (2), (4) and (5), the derivative of $S$ with respect to the time $t$ is derived as:

$$
\begin{aligned}
& \dot{S}=F(\boldsymbol{x})+\left(b(\boldsymbol{x})+\frac{\partial f_{1}(\boldsymbol{x})}{\partial x_{2}} b(\boldsymbol{x})\right) u_{a s t}+\hat{x}_{3} \\
& F(\boldsymbol{x})=f_{2}(\boldsymbol{x})+\frac{\partial f_{1}(\boldsymbol{x})}{\partial x_{1}}\left(x_{2}+f_{1}(\boldsymbol{x})\right)+\frac{\partial f_{1}(\boldsymbol{x})}{\partial x_{2}} f_{2}(\boldsymbol{x}) \\
& \quad+\varpi(t, T) \hbar_{2}\left(x_{1}-\hat{x}_{1}, T\right)+(1-\varpi(t, T)) \vartheta_{2}\left(x_{1}-\hat{x}_{1}\right)
\end{aligned}
$$

Hence, by defining $u_{\text {ast }}$ as:

$$
u_{a s t}=-\frac{F(\boldsymbol{x})+\hat{x}_{3}}{b(\boldsymbol{x})+\frac{\partial f_{1}(\boldsymbol{x})}{\partial x_{2}} b(\boldsymbol{x})}-\xi \tanh (S)
$$

we have $\dot{S}=0$, i.e., $S=0$ is guaranteed by the $u_{a s t}$ in Eq. (39). According to Assumption 3, $u_{\text {ast }}$ is continuous. Note that $\xi \tanh (S)$ is introduced to improve the control accuracy under the finite sampling frequency. In the continuous-time sense, $\xi \tanh (S)$ can be removed.

Accordingly, the sliding dynamics on the sliding surface $S=0$ read:

$\left\{\begin{array}{l}\dot{x}_{1}=\bar{x}_{2} \\ \dot{\bar{x}}_{2}=\left(b(\boldsymbol{x})+\frac{\partial f_{1}(\boldsymbol{x})}{\partial x_{2}} b(\boldsymbol{x})\right) u_{\text {norm }}(t)+\dot{\tilde{x}}_{2}\end{array}\right.$ 
Table 1 Parameter settings of Example 1

\begin{tabular}{lll}
\hline Methods & Detailed form & parameter settings \\
\hline Convergent form [14] & $\dot{V}=-\frac{1}{(1-\bar{p}) t_{f}} \frac{\kappa(V)^{\bar{p}}}{\kappa^{\prime}(V)}, \kappa(V)=\frac{V}{V+1}$ & $\bar{p}=0.5, t_{f}=5$ \\
Convergent form [27] & $\dot{V}=\left(e^{V}-1\right) \eta /\left[-e^{V}\left(t_{0}+t_{f}-t\right)\right]$ & $\eta=2, t_{f}=5$ \\
Proposed form (case 1) & $\dot{V}=\left(e^{V^{a}}-1\right) \eta /\left[-a V^{a-1} e^{V^{a}}\left(t_{0}+t_{f}-t\right)\right]$ & $\eta=2, t_{f}=5, a=0.5$ \\
Proposed form (case 2) & $\dot{V}=\left(e^{V^{a}}-1\right) \eta /\left[-a V^{a-1} e^{V^{a}}\left(t_{0}+t_{f}-t\right)\right]$ & $\eta=2, t_{f}=5, a=0.35$ \\
\hline
\end{tabular}

where $\tilde{x}_{2}$ denotes the observation error of $\bar{x}_{2}$. The remaining work in this subsection is to design $u_{\text {norm }}(t)$ for rendering the system (40) to the origin within a prescribed time interval.

Based on Theorem 2, the desired value of $\bar{x}_{2}$ is designed as:

$$
\begin{aligned}
& \bar{x}_{2 d}=-\varpi\left(t, t_{f 1}\right) \frac{\eta_{1}\left(e^{\sqrt{\overline{2}^{\bar{a}}}\left|x_{1}\right|^{\bar{a}}}-1\right) \operatorname{sgn}\left(x_{1}\right)}{\bar{a}\left|x_{1}\right|^{\bar{a}-1} e^{\sqrt{2^{a}}\left|x_{1}\right|^{\bar{a}}}\left(t_{0}+t_{f 1}-t\right)} \\
& -\left(1-\varpi\left(t, t_{f 1}\right)\right) x_{1}
\end{aligned}
$$

which can drive $x_{1}$ to zero within the time interval $\left[t_{0}, t_{0}+t_{f 1}\right)$ if is completely realized.

With the use of $\hat{x}_{2}$, we denote $z_{2}=\hat{x}_{2}-\bar{x}_{2 d}$ with its time-derivative expressed by:

$$
\begin{aligned}
& \dot{z}_{2}=\dot{\hat{x}}_{2}-\frac{\partial \bar{x}_{2 d}}{\partial t}-\bar{x}_{2} \frac{\partial \bar{x}_{2 d}}{\partial x_{1}} \\
& =\left(b(\boldsymbol{x})+\frac{\partial f_{1}(\boldsymbol{x})}{\partial x_{2}} b(\boldsymbol{x})\right) u_{n o r m}(t)-\frac{\partial \bar{x}_{2 d}}{\partial t}-\bar{x}_{2} \frac{\partial \bar{x}_{2 d}}{\partial x_{1}}
\end{aligned}
$$

where $\frac{\partial \bar{x}_{2 d}}{\partial x_{1}}$ and $\frac{\partial \bar{x}_{2 d}}{\partial t}$ represent the partial derivative of $\bar{x}_{2 d}$ with respect to $x_{1}$ and $t$, respectively.

$\left\{\begin{array}{l}\frac{\partial \bar{x}_{2 d}}{\partial t}=-\varpi\left(t, t_{f 1}\right) \frac{\eta_{1}\left(e^{\sqrt{\overline{2}^{\bar{a}}}\left|x_{1}\right|^{\bar{a}}}-1\right) \operatorname{sgn}\left(x_{1}\right)}{\bar{a}\left|x_{1}\right|^{\bar{a}-1} e^{\sqrt{2}^{a}}\left|x_{1}\right|^{\bar{a}}\left(t_{0}+t_{f 1}-t\right)^{2}} \\ \frac{\partial \bar{x}_{2 d}}{\partial x_{1}}=-\left(1-\varpi\left(t, t_{f 1}\right)\right) \\ -\varpi\left(t, t_{f 1}\right) \frac{\eta_{1}\left[\bar{a} \sqrt{2}{ }^{\bar{a}}\left|x_{1}\right|^{\bar{a}}-(\bar{a}-1)\left(e^{\left.\left.\sqrt{\overline{2}^{\bar{a}}}\left|x_{1}\right|^{\bar{a}}-1\right)\right]}\right.\right.}{\bar{a}\left(t_{0}+t_{f 1}-t\right)\left|x_{1}\right|^{\bar{a}} e^{\sqrt{\overline{2}^{a}}\left|x_{1}\right|^{\bar{a}}}}\end{array}\right.$

The design result of $u_{\text {norm }}(t)$ is given by:

$$
\begin{aligned}
& u_{\text {norm }}(t) \\
& =\frac{\left[\begin{array}{l}
-x_{1}+\frac{\partial \bar{x}_{2 d}}{\partial t}+\frac{\partial \bar{x}_{2 d}}{\partial x_{1}} \hat{x}_{2}-\left(1-\varpi\left(t, t_{f 1}\right)\right) z_{2} \\
-\varpi\left(t, t_{f 1}\right) \frac{\eta_{1}\left(e^{\sqrt{2}}\left|z_{2}\right|^{\bar{a}}\right.}{\bar{a}\left|z_{2}\right|^{\bar{a}-1} e^{\sqrt{2}}\left|z_{2}\right|^{\bar{a}}\left(t_{0}+t_{f 1}-t\right)}
\end{array}\right]}{b(\boldsymbol{x})+\frac{\partial f_{1}(\boldsymbol{x})}{\partial x_{2}} b(\boldsymbol{x})}
\end{aligned}
$$

At present, the CPTSMC has been constructed. The block diagram of the proposed control system is displayed in Fig. 3.

\subsubsection{Prescribed-time stability of the whole system}

In this subsection, we show the continuity and boundedness of the control law, the boundedness of the system signals, and the PTS of the system (1) under the drive of the control law. It should be stressed that the boundedness of the control law and system signals is vital for practical application and has not been explored in $[27,28]$; thus, it is deemed our contribution.

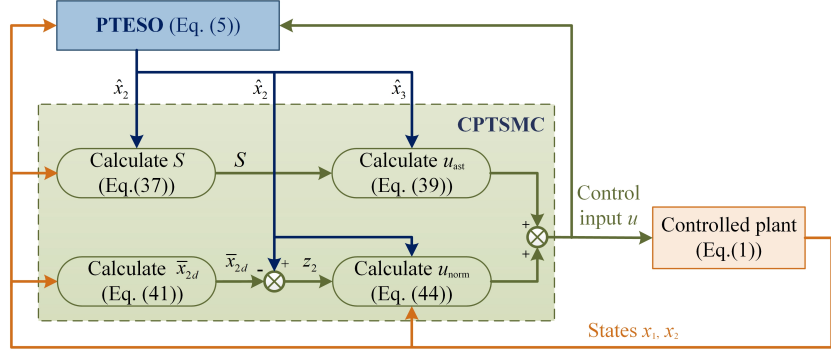

Fig. 3 Block diagram of the proposed control system

Using Eqs. (37), (40) and (44), the system (1) under the drive of $u(t)$ can be described by:

$\left\{\begin{array}{l}\dot{x}_{1}=\bar{x}_{2} \\ \dot{\bar{x}}_{2}=-x_{1}+\frac{\partial \bar{x}_{2 d}}{\partial t}+\frac{\partial \bar{x}_{2 d}}{\partial x_{1}} \hat{x}_{2}-\left(1-\varpi\left(t, t_{f 1}\right)\right) z_{2} \\ -\varpi\left(t, t_{f 1}\right) \frac{\eta_{1}\left(e^{\sqrt{\bar{a}^{a}}\left|z_{2}\right|^{a}}-1\right) \operatorname{sgn}\left(z_{2}\right)}{\bar{a}\left|z_{2}\right|^{\bar{a}-1} e^{\sqrt{2}^{a}\left|z_{2}\right|^{\bar{a}}}\left(t_{0}+t_{f 1}-t\right)}+\dot{\tilde{x}}_{2}\end{array}\right.$

Theorem 3 Let $\bar{a} \in(0,1), t_{f 1}>T$ with $T$ as the prescribed UBST of the PTESO, and $\eta_{1}>$ $\max \left\{\sqrt{2}^{2-\bar{a}}, 2 \bar{a} \sqrt{2}^{2-\bar{a}}\right\}$. For the system (1) under the drive of $u(t)$ in Eq. (37), $x_{1}$ and its time-derivative $\bar{x}_{2}$ converge to the origin within the prescribed time interval $\left[t_{0}, t_{0}+t_{f 1}\right)$. Moreover, $u(t)$ is bounded and continuous, and all system signals remain bounded.

Proof The proof is divided into four steps. The first step is the discussion on the convergence property within the time interval $\left[t_{0}, t_{0}+T\right)$, in which the observation errors of the PTESO have not reached zero. Then the convergence property of the system within the time interval $\left[t_{0}+T, t_{0}+t_{f 1}\right)$ is analysed in Step 2. Step 3 considers the condition within the time interval $\left[t_{0}+t_{f 1}, \infty\right)$. The analysis on the boundedness of system signals and the continuity of $u(t)$ is elaborated in Step 4.

Step 1: Within the time interval $\left[t_{0}, t_{0}+T\right)$, given that $\bar{x}_{2}=z_{2}+\bar{x}_{2 d}+\tilde{x}_{2}$, the system of $x_{1}$ and $z_{2}$ is depicted by:

$$
\left\{\begin{array}{l}
\dot{x}_{1}=z_{2}-\frac{\eta_{1}\left(e^{\sqrt{2^{\bar{a}}}\left|x_{1}\right|^{\bar{a}}}-1\right) \operatorname{sgn}\left(x_{1}\right)}{\bar{a}\left|x_{1}\right|^{\bar{a}-1} e^{\sqrt{\bar{a}^{a}}\left|x_{1}\right|^{\bar{a}}}\left(t_{0}+t_{f 1}-t\right)}+\tilde{x}_{2} \\
\dot{z}_{2}=-x_{1}-\frac{\partial \bar{x}_{2 d}}{\partial x_{1}} \tilde{x}_{2}-\frac{\eta_{1}\left(e^{\sqrt{\overline{2}^{a}}\left|z_{2}\right|^{\bar{a}}}-1\right) \operatorname{sgn}\left(z_{2}\right)}{\bar{a}\left|z_{2}\right|^{\bar{a}-1} e^{\sqrt{\bar{a}^{a}}\left|z_{2}\right|^{\bar{a}}}\left(t_{0}+t_{f 1}-t\right)}
\end{array}\right.
$$


By taking the Lyapunov candidate function $V_{1}=$ $x_{1}^{2}+z_{2}^{2}$, the time-derivative of $V_{1}$ is:

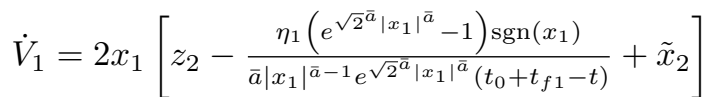

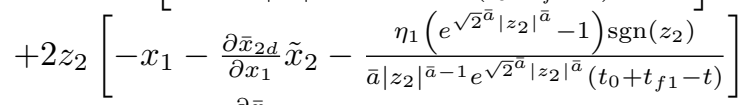

$$
\begin{aligned}
& \leq 2 x_{1} \tilde{x}_{2}-2 z_{2} \frac{\partial \bar{x}_{2 d}}{\partial x_{1}} \tilde{x}_{2}
\end{aligned}
$$

As shown in the proof of Theorem 1, the observation error $\tilde{x}_{2}$ remains bounded within the time interval $\left[t_{0}, t_{0}+T\right)$. Regarding the item $\partial \bar{x}_{2 d} / \partial x_{1}$, of which the detailed form is presented in Eq. (43). By using the L'Hôpital's rule [48], there is:

$$
\begin{aligned}
& \lim _{x_{1} \rightarrow 0} \frac{\partial \bar{x}_{2 d}}{\partial x_{1}} \\
& =-\lim _{x_{1} \rightarrow 0} \frac{\eta_{1}\left[\bar{a} \sqrt{2} \bar{a}^{\bar{a}}\left|x_{1}\right|^{\bar{a}}-(\bar{a}-1)\left(e^{\sqrt{2^{\bar{a}}}\left|x_{1}\right|^{\bar{a}}}-1\right)\right]}{\bar{a}\left(t_{0}+t_{f 1}-t\right)\left|x_{1}\right|^{\bar{a}} e^{\sqrt{2^{a}}\left|x_{1}\right|^{\bar{a}}}} \\
& =\frac{-\sqrt{2}^{\bar{a}} \eta_{1}}{\bar{a}\left(t_{0}+t_{f 1}-t\right)}
\end{aligned}
$$

Hence, $\partial \bar{x}_{2 d} / \partial x_{1}$ remains bounded as $x_{1}$ approaches zero within the time interval $\left[t_{0}, t_{0}+T\right)$.

Define $M=\sup _{t \in\left[t_{0}, t_{0}+T\right)}\left\{\left|\tilde{x}_{2}\right|,\left|\tilde{x}_{2} \partial \bar{x}_{2 d} / \partial x_{1}\right|\right\}$. With Eq. (47), it can be obtained that $\dot{V}_{1} \leq 4 M V_{1}^{0.5}$, which means $V_{1}$ will not escape to the infinity within $\left[t_{0}, t_{0}+T\right)$ considering the small value of $T$.

Step 2: Within the time interval $\left[t_{0}+T, t_{0}+t_{f 1}\right)$, ascribed to the PTS of the proposed ESO, the system of $x_{1}$ and $z_{2}$ changes into:

$$
\left\{\begin{array}{l}
\dot{x}_{1}=z_{2}-\frac{\eta_{1}\left(e^{\sqrt{2^{\bar{a}}}\left|x_{1}\right|^{\bar{a}}}-1\right) \operatorname{sgn}\left(x_{1}\right)}{\bar{a}\left|x_{1}\right|^{\bar{a}-1} e^{\sqrt{2} a}\left|x_{1}\right|^{\bar{a}}\left(t_{0}+t_{f 1}-t\right)} \\
\dot{z}_{2}=-\frac{\eta_{1}\left(e^{\sqrt{2} \bar{a}}\left|z_{2}\right|^{\bar{a}}-1\right) \operatorname{sgn}\left(z_{2}\right)}{\bar{a}\left|z_{2}\right|^{\bar{a}-1} e^{\sqrt{2} \bar{a}^{a}\left|z_{2}\right|^{\bar{a}}}\left(t_{0}+t_{f 1}-t\right)}-x_{1}
\end{array}\right.
$$

Accordingly, the time-derivative of $V_{1}=x_{1}^{2}+z_{2}^{2}$ becomes:

$$
\begin{aligned}
& \dot{V}_{1}=2 x_{1} \dot{x}_{1}+2 z_{2} \dot{z}_{2} \\
& =-2 \frac{\eta_{1}\left(e^{\sqrt{2^{\bar{a}}}\left|x_{1}\right|^{\bar{a}}}-1\right)\left|x_{1}\right|}{\bar{a}\left|x_{1}\right|^{\bar{a}-1} e^{\sqrt{2} \bar{a}}\left|x_{1}\right|^{\bar{a}}\left(t_{0}+t_{f 1}-t\right)} \\
& -2 \frac{\eta_{1}\left(e^{\sqrt{2} \bar{a}^{\bar{a}}\left|z_{2}\right|^{\bar{a}}}-1\right)\left|z_{2}\right|}{\bar{a}\left|z_{2}\right|^{\bar{a}-1} e^{\sqrt{2^{a}}\left|z_{2}\right|^{\bar{a}}}\left(t_{0}+t_{f 1}-t\right)}
\end{aligned}
$$

Denote $Q=\max \left\{\left|x_{1}\right|,\left|z_{2}\right|\right\}$. Obviously, $\frac{\sqrt{2 V_{1}}}{2} \leq$ $Q \leq \sqrt{V_{1}}$. Therefore, it holds that:

$0 \leq \frac{e^{\sqrt{V_{1}} \bar{a}}-1}{e^{{\sqrt{V_{1}}}^{a}}} \leq \frac{e^{\sqrt{\overline{2}^{\bar{a}}} Q^{\bar{a}}}-1}{e^{\sqrt{2} \bar{a}} Q^{\bar{a}}}$

$\Rightarrow \dot{V}_{1} \leq-2 \frac{\eta_{1}\left(e^{\sqrt{\overline{2}^{\bar{a}}} Q^{\bar{a}}}-1\right) Q^{2-\bar{a}}}{\bar{a} e^{\sqrt{\overline{2}^{a}} Q^{\bar{a}}}\left(t_{0}+t_{f 1}-t\right)}$

$\leq-2 \frac{\eta_{1}\left(e^{{\sqrt{V_{1}}}^{\bar{a}}}-1\right)\left(\sqrt{2 V_{1}} / 2\right)^{2-\bar{a}}}{\bar{a} e^{{\sqrt{V_{1}}}^{\bar{a}}}\left(t_{0}+t_{f 1}-t\right)}$

$\leq-2\left(\frac{\sqrt{2}}{2}\right)^{2-\bar{a}} \frac{\eta_{1}\left(e^{\sqrt{V_{1}}-\bar{a}}-1\right)\left(\sqrt{V_{1}}\right)^{2-\bar{a}}}{\bar{a} e^{\sqrt{V_{1}} \bar{a}}\left(t_{0}+t_{f 1}-t\right)}$

$\Rightarrow \frac{\dot{V}_{1}}{2 \sqrt{V_{1}}} \leq-\left(\frac{\sqrt{2}}{2}\right)^{2-\bar{a}} \frac{\eta_{1}\left(e^{\sqrt{V_{1}} \bar{a}}-1\right)}{\bar{a} e^{{\sqrt{V_{1}}}^{\bar{a}}}\left(t_{0}+t_{f 1}-t\right) \sqrt{V_{1}} \bar{a}-1}$
By defining $\Lambda=\sqrt{V_{1}}$, Eq. (51) is converted into:

$$
\dot{\Lambda} \leq-\left(\frac{\sqrt{2}}{2}\right)^{2-\bar{a}} \frac{\eta_{1}\left(e^{\Lambda^{\bar{a}}}-1\right)}{\bar{a} e^{\Lambda^{\bar{a}}}\left(t_{0}+t_{f 1}-t\right) \Lambda^{\bar{a}-1}}
$$

According to Theorem 2 , if $\eta_{1}>\sqrt{2}^{2-\bar{a}}, \Lambda$ converges to zero within the time interval $\left[t_{0}+T, t_{0}+t_{f 1}\right)$. In other words, $x_{1}$ and $z_{2}$ converge to zero within the time interval $\left[t_{0}+T, t_{0}+t_{f 1}\right)$.

However, it is difficult to directly obtain the value of $\bar{x}_{2}$ at the switch time $t=t_{0}+t_{f 1}$ due to the complicated form of $\bar{x}_{2 d}$. To analyse $\bar{x}_{2}$, the following inequality that always holds for $\bar{a} \in(0,1)$ is used:

$$
\frac{e^{\sqrt{2^{\bar{a}}}\left|x_{1}\right|^{\bar{a}}}-1}{\bar{a}\left|x_{1}\right|^{\bar{a}-1} e^{\sqrt{2^{\bar{a}}}\left|x_{1}\right|^{\bar{a}}}} \leq \frac{e^{\sqrt{2^{\bar{a}}} \Lambda^{\bar{a}}}-1}{\bar{a} \Lambda^{\bar{a}-1} e^{\sqrt{2^{\bar{a}}}\left|x_{1}\right|^{\bar{a}}}}
$$

Considering Eq. (52), by integrating double sides over time, the trajectory of $\Lambda$ is obtained:

$\Lambda(t) \leq\left[\ln \left(C_{1}\left(t_{0}+t_{f 1}-t\right)^{\sqrt{2}^{\bar{a}-2} \eta_{1}}+1\right)\right]^{1 / \bar{a}}$

where $C_{1}=\left(e^{\Lambda\left(t_{0}+T\right)^{\bar{a}}}-1\right) /\left(t_{f_{1}}-T\right)^{\sqrt{2}^{\bar{a}-2} \eta_{1}}$.

Based on Eqs. (53), (54) and L'Hôpital's rule [48], there is:

$$
\begin{aligned}
& \left|\bar{x}_{2 d}\right|=\frac{\eta_{1}\left(e^{\sqrt{\overline{2}^{\bar{a}}}\left|x_{1}\right|^{\bar{a}}}-1\right)}{\bar{a}\left|x_{1}\right|^{\bar{a}-1} e^{\sqrt{\overline{2}^{\bar{a}}}\left|x_{1}\right|^{\bar{a}}}\left(t_{0}+t_{f 1}-t\right)} \\
& \leq \frac{\eta_{1}\left(e^{\sqrt{2^{\bar{a}}} \Lambda^{\bar{a}}}-1\right)}{\bar{a} \Lambda^{\bar{a}-1} e^{\sqrt{\overline{2}^{\bar{a}}}\left|x_{1}\right|^{\bar{a}}\left(t_{0}+t_{f 1}-t\right)}} \\
& \leq \frac{\left(\eta_{1} e^{\Phi}-\eta_{1}\right)\left(\ln \left(C_{1}\left(t_{f 1}-t\right)^{\sqrt{2} \bar{a}-2} \eta_{1}+1\right)\right)^{(1-\bar{a}) / \bar{a}}}{\bar{a} e^{\sqrt{2^{a}}\left|x_{1}\right|^{\bar{a}}}\left(t_{0}+t_{f 1}-t\right)} \\
& \stackrel{L^{\prime} H o p i t a l^{\prime} \text { s rule }}{\Rightarrow} \lim _{t \rightarrow t_{0}+t_{f 1}} \frac{\eta_{1}\left(e^{\sqrt{\overline{2}^{\bar{a}}}\left|x_{1}\right|^{\bar{a}}}-1\right)}{\bar{a}\left|x_{1}\right|^{\bar{a}-1} e^{\sqrt{\overline{2}^{\bar{a}}}\left|x_{1}\right|^{\bar{a}}}\left(t_{0}+t_{f 1}-t\right)} \leq 0 \\
& \Rightarrow \lim _{t \rightarrow t_{0}+t_{f 1}} \bar{x}_{2 d}=0
\end{aligned}
$$

where $\Phi=\sqrt{2}^{\bar{a}} \ln \left[C_{1}\left(t_{0}+t_{f 1}-t\right)^{\sqrt{2}^{\bar{a}-2} \eta_{1}}+1\right]$. Thus, recalling $\bar{x}_{2}=z_{2}+\bar{x}_{2 d}+\tilde{x}_{2}, \bar{x}_{2}$ tends to be zero as $t \rightarrow$ $t_{0}+t_{f 1}$. With regard to the system (1), if $f_{1}(\boldsymbol{x})+d_{1}(t)$ remains bounded, then the state $x_{2}$ is bounded.

Step 3: Within the time interval $\left[t_{0}+t_{f 1}, \infty\right)$, the system of $x_{1}$ and $z_{2}$ is:

$\left\{\begin{array}{l}\dot{x}_{1}=z_{2}-x_{1} \\ \dot{z}_{2}=-z_{2}-x_{1}\end{array}\right.$

Note that the value of $\bar{x}_{2 d}\left(t_{0}+t_{f 1}\right)$ equals 0 and is not changed by the switch action. Therefore, $x_{1}\left(t_{0}+t_{f 1}\right)=0$ and $z_{2}\left(t_{0}+t_{f 1}\right)=0$ are valid for system (56), and $\left(x_{1}, z_{2}\right)$ stays at the origin thereafter.

Step 4: In this step, the boundedness of system signals and continuity of $u(t)$ are discussed. Recalling $u(t)=u_{\text {norm }}(t)+u_{\text {ast }}(t)$, in which $u_{\text {ast }}(t)$ in Eq. (39) is definitely bounded and continuous. In addition, according to Eq. (44), it is clear that $u_{\text {norm }}(t)$ is bounded 
within the time interval $\left[t_{0}+t_{f 1}, \infty\right)$. For the time interval $\left[t_{0}, t_{0}+T\right)$, considering the boundedness of $\partial \bar{x}_{2 d} / \partial x_{1}$ in Eq. (48), $u_{\text {norm }}(t)$ also remains bounded. Hence, the condition within the time interval $\left[t_{0}+T, t_{0}+t_{f 1}\right)$ is the focus, under which the trajectories of $x_{1}$ and $z_{2}$ follow:

$$
\begin{aligned}
& \left|x_{1}\right| \leq \Lambda(t) \leq\left[\ln \left(C_{1}\left(t_{0}+t_{f 1}-t\right)^{\sqrt{2}^{\bar{a}-2} \eta_{1}}+1\right)\right]^{\frac{1}{\bar{a}}} \\
& \left|z_{2}\right| \leq \Lambda(t) \leq\left[\ln \left(C_{1}\left(t_{0}+t_{f 1}-t\right)^{\sqrt{2}^{\bar{a}-2} \eta_{1}}+1\right)\right]^{\frac{1}{\bar{a}}}
\end{aligned}
$$

For convenience of the expression, we define:

$u_{n A}=\frac{\eta_{1}\left(e^{\sqrt{2^{\bar{a}}}\left|z_{2}\right|^{\bar{a}}}-1\right)}{\bar{a}\left|z_{2}\right|^{\bar{a}-1} e^{\sqrt{2}^{\bar{a}}\left|z_{2}\right|^{\bar{a}}}\left(t_{0}+t_{f 1}-t\right)}$

$u_{n B}=\frac{\partial \bar{x}_{2 d}}{\partial t}+\frac{\partial \bar{x}_{2 d}}{\partial x_{1}} \hat{x}_{2}$

Given that $u_{n A}$ is nonnegative and increases along with $\left|z_{2}\right|$, by defining $\tilde{\Lambda}=$ $\left[\ln \left(C_{1}\left(t_{0}+t_{f 1}-t\right)^{\sqrt{2}^{\bar{a}-2} \eta_{1}}+1\right)\right]^{1 / \bar{a}}$, we have:

$$
\begin{aligned}
& u_{n A} \leq \frac{\eta_{1}\left(e^{\sqrt{\overline{2}^{\bar{a}}} \tilde{\Lambda}^{\bar{a}}}-1\right)}{\bar{a} e^{\sqrt{\overline{2}^{\bar{a}}} \tilde{\Lambda}^{\bar{a}}\left(t_{0}+t_{f 1}-t\right) \tilde{\Lambda}^{\bar{a}-1}}} \\
& \Rightarrow \lim _{t \rightarrow t_{0}+t_{f 1}} u_{n A} \\
& \leq \lim _{t \rightarrow t_{0}+t_{f 1}} \frac{\eta_{1}\left(e^{\sqrt{2} \bar{a} \tilde{\Lambda}^{\bar{a}}}-1\right)}{\bar{a} e^{\sqrt{2} \bar{a}} \tilde{\Lambda}^{\bar{a}}\left(t_{0}+t_{f 1}-t\right) \tilde{\Lambda}^{\tilde{a}-1}}=0 \\
& \Rightarrow \lim _{t \rightarrow t_{0}+t_{f 1}} u_{n A}=0
\end{aligned}
$$

Hence, $u_{n A}$ is a bounded term within $\left[t_{0}+T, t_{0}+t_{f 1}\right)$.

The remaining work is about the property of $u_{n B}$. As indicated in Eq. (43), $\left|\partial \bar{x}_{2 d} / \partial t\right|$ is positively correlated with $\left|x_{1}\right|$. The limit value of $\left|\partial \bar{x}_{2 d} / \partial t\right|$ as $t \rightarrow$ $t_{0}+t_{f 1}$ satisfies:

$$
\lim _{t \rightarrow t_{0}+t_{f 1}}\left|\frac{\partial \bar{x}_{2 d}}{\partial t}\right| \leq \lim _{t \rightarrow t_{0}+t_{f 1}} \frac{\eta_{1}\left(e^{\sqrt{2^{\bar{a}}} \tilde{\Lambda}^{\bar{a}}}-1\right) \tilde{\Lambda}^{1-\bar{a}}}{\bar{a}\left(t_{0}+t_{f 1}-t\right)^{2}}
$$

Following the L'Hôpital's rule [48], there is:

$$
\begin{aligned}
& \lim _{t \rightarrow t_{0}+t_{f 1}} \frac{\left(e^{\sqrt{2}^{\bar{a}} \tilde{\Lambda}^{\bar{a}}}-1\right) \tilde{\Lambda}^{1-\bar{a}}}{\left(t_{0}+t_{f 1}-t\right)^{2}} \\
& =\lim _{t \rightarrow t_{0}+t_{f 1}} \dot{\tilde{\Lambda}} \frac{(1-\bar{a}) \tilde{\Lambda}^{-\bar{a}}\left(e^{\sqrt{2} \bar{a}} \tilde{\Lambda}^{\bar{a}}-1\right)+\bar{a} \sqrt{2^{\bar{a}}} e^{\sqrt{2^{\bar{a}}} \tilde{\Lambda}^{\bar{a}}}}{-2\left(t_{0}+t_{f 1}-t\right)} \\
& =\lim _{t \rightarrow t_{0}+t_{f 1}} \frac{\sqrt{2}^{\bar{a}} \dot{\tilde{\Lambda}}}{-2\left(t_{0}+t_{f 1}-t\right)}
\end{aligned}
$$

Plugging $\tilde{\Lambda}=\left[\ln \left(C_{1}\left(t_{0}+t_{f 1}-t\right)^{\sqrt{2}^{\bar{a}-2} \eta_{1}}+1\right)\right]^{\frac{1}{\bar{a}}}$ into Eq. (62) and further simplification yield:

$$
\begin{aligned}
& \lim _{t \rightarrow t_{0}+t_{f 1}} \frac{\left(e^{\sqrt{2^{\bar{a}}} \tilde{\Lambda}^{\bar{a}}}-1\right) \tilde{\Lambda}^{1-\bar{a}}}{\left(t_{0}+t_{f 1}-t\right)^{2}} \\
& =\lim _{t \rightarrow t_{0}+t_{f 1}}\left\{\begin{array}{l}
\left\{\frac{\ln \left[C_{1}\left(t_{0}+t_{f 1}-t\right)^{\sqrt{2} \bar{a}-2} \eta_{1}+1\right]}{\left(t_{0}+t_{f 1}-t\right)^{\sqrt{2}^{\bar{a}-2} \eta_{1}}}\right\}^{\frac{(1-\bar{a})}{\bar{a}}} \\
\times \frac{\sqrt{2}^{2 \bar{a}-2} \eta_{1} C_{1}\left(t_{0}+t_{f 1}-t\right) \frac{\sqrt{2}^{\bar{a}-2} \eta_{1}}{\bar{a}}-1}{2 \bar{a}\left(t_{0}+t_{f 1}-t\right)}
\end{array}\right\} \\
& =\frac{\sqrt{2}^{2 \bar{a}-2} \eta_{1} C_{1}\left(t_{0}+t_{f 1}-t\right) \frac{\sqrt{2}^{\bar{a}-2} \eta_{1}}{\bar{a}}-1}{2 \bar{a}\left(t_{0}+t_{f 1}-t\right)}
\end{aligned}
$$

Hence, if $\eta_{1}>2 \bar{a} \sqrt{2}^{2-\bar{a}}, \partial \bar{x}_{2 d} / \partial t$ tends to be 0 as $t \rightarrow$ $t_{0}+t_{f 1}$ and remains bounded within $\left[t_{0}+T, t_{0}+t_{f 1}\right)$.

Regarding the item $\hat{x}_{2} \partial \bar{x}_{2 d} / \partial \bar{x}_{1}$ in $u_{n B}$, it follows:

$$
\begin{aligned}
& \left|\hat{x}_{2} \partial \bar{x}_{2 d} / \partial x_{1}\right| \leq\left(\left|z_{2}\right|+\left|\bar{x}_{2 d}\right|\right) \times\left|\partial \bar{x}_{2 d} / \partial x_{1}\right|
\end{aligned}
$$

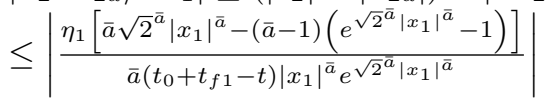

$$
\begin{aligned}
& \times\left\{\begin{array}{l}
{\left[\ln \left(C_{1}\left(t_{0}+t_{f 1}-t\right)^{\sqrt{2} \bar{a}-2} \eta_{1}+1\right)\right]^{1 / \bar{a}}} \\
+\frac{\eta_{1}\left(e^{\sqrt{2} \bar{a}\left|x_{1}\right|^{\bar{a}}}-1\right)}{\bar{a}\left|x_{1}\right|^{\bar{a}-1} e^{\sqrt{2} a}\left|x_{1}\right|^{\bar{a}}\left(t_{0}+t_{f 1}-t\right)}
\end{array}\right\}
\end{aligned}
$$

Likewise, through utilizing L'Hôpital's rule, it is easy to find that $\lim _{t \rightarrow t_{0}+t_{f 1}}\left|\hat{x}_{2} \partial x_{2 d} / \partial x_{1}\right|=0$. Hence, $\hat{x}_{2} \partial \bar{x}_{2 d} / \partial x_{1}$ remains bounded within $\left[t_{0}+T, t_{0}+t_{f 1}\right)$.

At this point, the boundedness of $u_{n A}$ and $u_{n B}$ within $\left[t_{0}+T, t_{0}+t_{f 1}\right)$ has been proved. By invoking Eq. (44), the boundedness of $u_{n o r m}(t)$ has been proved. Equivalently, $u(t)$ remains bounded. Based on Eqs. (37), (52) and (55), the conclusion is valid that all system signals $\left\{S, u, x_{1}, x_{2}, z_{2}, \bar{x}_{2 d}\right\}$ are bounded. Furthermore, $u_{\text {norm }}\left(t_{0}+t_{f 1}\right)=0$ holds true regardless of the switch action, which means that the continuity of $u_{\text {norm }}(t)$ is not broken at $t=t_{0}+t_{f 1}$. Based on the continuity of $u_{a s t}(t)$, it can be concluded that $u(t)$ is continuous. This completes the proof of Theorem 3 .

Remark 9 The UBST of system (1) under the drive of CPTSMC is prescribed by the parameter $t_{f 1}$ and irrelevant to initial conditions. Compared with the FTSMC and FxTSMC [7-12], the tuning rules of the CPTSMC are substantially simplified. Regarding the PTSMC [15-18], the CPTSMC is much less conservative in meeting the temporal demands, as shown in Theorem 2. Furthermore, due to continuity, the CPTSMC enjoys the chattering-alleviated convergence process compared to the PTSMC [15-18,27-30] under the finite sampling frequency. Although the PTSMC laws [27, 28] also exhibit weak conservativeness, some issues remain to be settled due to its aggressive convergence, such as the accuracy loss shown in Fig. 2. Most importantly, we show the boundedness of system signals, which has not been proved in $[27,28]$. Considering the saturation constraints (the allowed range of $u(t)$ ) and velocity constraints (the allowed range of $\dot{x}_{1}$ ) $[49,50]$, the boundedness of system signals is necessary and meaningful.

Remark 10 (Parameter tuning advice) As shown in Theorem 3, parameter tuning rules of the CPTSMC for temporal demands are considerably simple. After selecting $\bar{a}$ and $\eta_{1}$ following Theorem 3, different temporal demands can be met by simply tuning $t_{f 1}$. Considering the saturation constraints, the setting of $t_{f 1}$ is exactly a trade-off between the control capability and convergence rate. It should be stressed that even when 
saturation occurs, the prescribed UBST $t_{f 1}$ can be realized once saturation disappears before $t=t_{0}+t_{f 1}$ (the system stability under saturation is not within the scope of this paper). Although the boundedness of the control input has been validated theoretically, infinite control input may occur due to the finite sampling frequency and measurement noise in practical applications. Hence, it is advisable to implement the switch slightly earlier than $t=t_{0}+t_{f 1}$ to avoid the possible infinite control input. To compensate for the accuracy loss caused by the early switch, a large $\eta_{1}$ should be chosen such that $x_{1}$ can reach the small neighbourhood of the origin at a faster rate (this conclusion can be easily obtained through Eq. (52)). Furthermore, when oscillation occurs due to the large $\dot{x}_{1}$ near $t=t_{0}+t_{f 1}$, turning down the value of $\bar{a}$ is a correct choice.

\section{Simulation and analysis}

In this section, numerical simulations are presented to illustrate the efficiency of our method. In Subsection 4.1, the PTESO is compared with the existing ESOs to validate its PTS, weak conservativeness and muchreduced peaking values. Subsection 4.2 makes the comparison between the CPTSMC and the PTSMC methods $[14,27]$ to verify the weak conservativeness and high transient performance of the CPTSMC. In Subsection 4.3, a DC servo system under time-varying mismatched disturbances is selected to highlight the practical application values of the proposed control system.

\subsection{Simulation of the PTESO}

Consider a second-order system $\dot{x}_{1}=x_{2}+d_{1}(t), \dot{x}_{2}=$ $d_{2}(t)+u(t) \cdot u(t)$ is set as zero. $d_{1}(t)=\sin (2 t)$ and $d_{2}(t)=2.5 \cos (2 t)-29.5$ are unmeasurable disturbances. The initial values are $x_{1}\left(t_{0}\right)=5$ and $x_{2}\left(t_{0}\right)=$ -15 . By regarding the second-order system as a thirdorder system in the form of Eq. (4), we have $\bar{x}_{2}=$ $x_{2}+d_{1}(t)$ and $\bar{x}_{3}=\dot{d}_{1}(t)+d_{2}(t)$, of which the initial values should be $\bar{x}_{2}\left(t_{0}\right)=-15$ and $\bar{x}_{3}\left(t_{0}\right)=-25$. The simulation step size is $1 \mathrm{e}-3 \mathrm{~s}$. The initial time $t_{0}=0 \mathrm{~s}$.

Simulation A: To validate the PTS of the PTESO, four cases are adopted with $T=0.8, T=1.2, T=1.6$, and $T=2$. Other parameter settings are: $\iota_{1}=0.1, \iota_{2}=$ $0.3, \iota_{3}=0.09, \nu=1, k_{1}=3.823, k_{2}=6.945, k_{3}=1.5$, and $L=10$. Since $x_{1}$ can be measured, we focus on the observation efficiency of $\bar{x}_{2}$ and $\bar{x}_{3}$. Simulation results are shown in Figs. 4 and 5.

The simulation results show that the precise estimation of $\bar{x}_{2}$ and $\bar{x}_{3}$ has been realized with UBST $T$ in four cases. For $T=0.8, \tilde{x}_{2}(T)=-4.8 \mathrm{e}-5, \tilde{x}_{3}(T)=-0.068$.
For $T=1.2, \tilde{x}_{2}(T)=-9.9 \mathrm{e}-6, \tilde{x}_{3}(T)=-0.041$. For $T=1.6, \tilde{x}_{2}(T)=-1.6 \mathrm{e}-5, \tilde{x}_{3}(T)=0.0018$. For $T=2$, $\tilde{x}_{2}(T)=1.2 \mathrm{e}-5, \tilde{x}_{3}(T)=0.0043$. Furthermore, shown by Figs. 4 and 5 , the actual settling time of observation errors $\tilde{x}_{2}$ and $\tilde{x}_{3}$ is approximate to $T$ in all simulation cases, indicating the weak conservativeness in prescribing the UBST. Therefore, by simply tuning the value of $T$, different observation temporal demands can be met tightly. Moreover, Figs. 4 and 5 present the considerably small overshoot of the observation errors $\tilde{x}_{2}$ and $\tilde{x}_{3}$ in four simulation cases, which is desired since $\hat{x}_{2}$ and $\hat{x}_{3}$ are leveraged in the CPTSMC. As analysed in Subsection 3.1, the high transient performance is ascribed to the monotone increasing function $\mu_{1}(t)$, which has a small initial value and grows to infinity as $t \rightarrow t_{0}+T$.

Simulation B: For contrast, the linear ESO [51], finite-time ESO [41] and fixed-time ESO [38] are chosen, which are respectively written as 'LESO', 'FTESO' and 'FxTESO'. With $\tilde{x}_{1}=x_{1}-\hat{x}_{1}$, three ESOs are given as follows:

$$
\begin{gathered}
\text { LESO : }\left\{\begin{array}{l}
\dot{\hat{x}}_{1}=\hat{x}_{2}+3 \omega_{0} \tilde{x}_{1} \\
\hat{\hat{x}}_{2}=\hat{x}_{3}+3 \omega_{0}^{2} \tilde{x}_{1} \\
\dot{\hat{x}}_{3}=\omega_{0}^{3} \tilde{x}_{1}
\end{array}\right. \\
\text { FTESO }:\left\{\begin{array}{l}
\dot{\hat{x}}_{1}=\hat{x}_{2}+\bar{k}_{1}\left\lceil\tilde{x}_{1}\right\rfloor^{\bar{\alpha}_{1}} \\
\dot{\hat{x}}_{2}=\hat{x}_{3}+\bar{k}_{2}\left\lceil\tilde{x}_{1}\right]^{\bar{\alpha}_{2}} \\
\dot{\hat{x}}_{3}=\bar{k}_{3}\left\lceil\tilde{x}_{1}\right\rfloor^{\bar{\alpha}_{3}}
\end{array}\right.
\end{gathered}
$$

FxTESO :

$\left\{\begin{array}{l}\dot{\hat{x}}_{1}=\hat{x}_{2}+v \tilde{k}_{1}\left(\left\lceil\frac{\tilde{x}_{1}}{v^{2}}\right\rfloor^{\tilde{\alpha}_{1}}+\left\lceil\frac{\tilde{x}_{1}}{v^{2}}\right\rfloor+\left\lceil\frac{\tilde{x}_{1}}{v^{2}}\right\rfloor^{\tilde{\beta}_{1}}\right) \\ \dot{\hat{x}}_{2}=\hat{x}_{3}+\tilde{k}_{2}\left(\left\lceil\frac{\tilde{x}_{1}}{v^{2}}\right\rfloor^{\tilde{\alpha}_{2}}+\left\lceil\frac{\tilde{x}_{1}}{v^{2}}\right\rfloor+\left\lceil\frac{\tilde{x}_{1}}{v^{2}}\right\rfloor^{\tilde{\beta}_{2}}\right) \\ \dot{\hat{x}}_{3}=\frac{\tilde{k}_{3}}{v}\left(\left\lceil\frac{\tilde{x}_{1}}{v^{2}}\right\rfloor^{\tilde{\alpha}_{3}}+\left\lceil\frac{\tilde{x}_{1}}{v^{2}}\right\rfloor+\left\lceil\frac{\tilde{x}_{1}}{v^{2}}\right\rfloor^{\tilde{\beta}_{3}}\right)\end{array}\right.$

In the simulation, the initial values of ESOs are chosen as $\hat{x}_{1}\left(t_{0}\right)=5, \hat{x}_{2}\left(t_{0}\right)=0$, and $\hat{x}_{3}\left(t_{0}\right)=0$. For the reasonability of the comparison, the desired settling time of observation errors is set as $0.6 \mathrm{~s}$, and the parameter settings of different ESOs are accordingly tuned. The tuning results are shown in Table. 2, and the simulation results are displayed in Figs. 6 and 7 .

As shown in Figs. 6 and 7, the proposed PTESO realizes the precise observation of $\bar{x}_{2}$ and $\bar{x}_{3}$ within $\left[t_{0}, t_{0}+T\right)$. The values of $\tilde{x}_{2}\left(t_{0}+T\right)$ and $\tilde{x}_{3}\left(t_{0}+T\right)$ are $6.87 \mathrm{e}-5$ and 0.01787 , respectively. Although the actual settling time of FTESO and FxTESO is approximately $0.6 \mathrm{~s}$, their estimated UBST is far larger than 0.6 s. Furthermore, it is not easy to find a positivedefinite matrix to calculate the UBST of FTESO and FxTESO, as shown in $[38,41]$. In contrast, the UBST of the PTESO is precisely prescribed by only one param- 
Table 2 Parameter settings of different ESOs

\begin{tabular}{ll}
\hline Methods & parameter settings \\
\hline LESO [51] & $\omega_{0}=20$ \\
FTESO [41] & $\bar{k}_{1}=100, \quad \bar{k}_{2}=500, \quad \bar{k}_{3}=1500, \quad \bar{\alpha}_{1}=0.9, \quad \bar{\alpha}_{2}=0.8, \quad \bar{\alpha}_{3}=0.7$ \\
FxTESO [38] & $v=0.3, \tilde{k}_{1}=20, \tilde{k}_{2}=100, \tilde{k}_{3}=500, \tilde{\alpha}_{1}=0.95, \tilde{\alpha}_{2}=0.9, \tilde{\alpha}_{3}=0.85, \tilde{\beta}_{1}=1.05, \tilde{\beta}_{2}=1.1, \tilde{\beta}_{3}=1.15$ \\
PTESO & $\iota_{1}=0.1, \iota_{2}=0.3, \iota_{3}=0.09, T=0.6, \nu=1, \quad k_{1}=4.2, k_{2}=8.42, \quad k_{3}=2, L=10$ \\
\hline
\end{tabular}

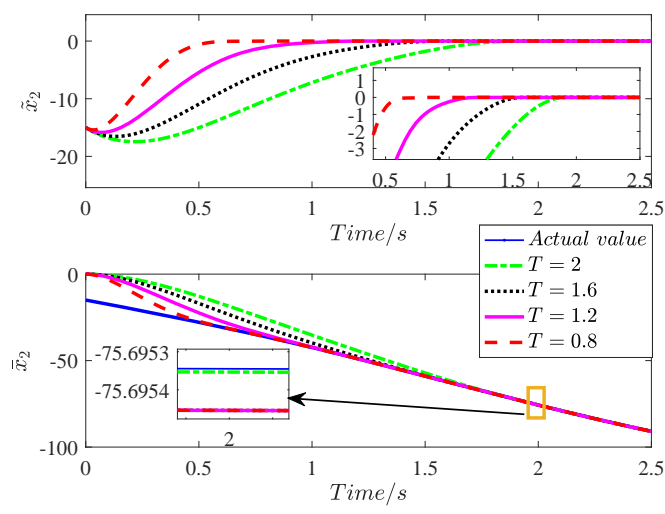

Fig. 4 Time histories of $\bar{x}_{2}$ and $\tilde{x}_{2}$ in Simulation A

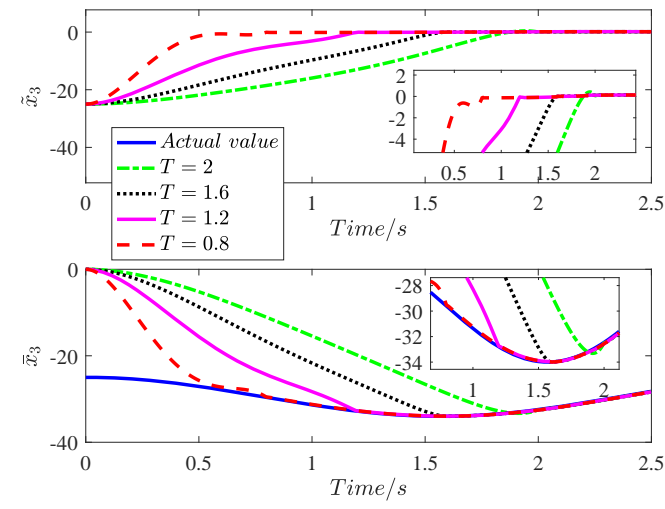

Fig. 5 Time histories of $\bar{x}_{3}$ and $\tilde{x}_{3}$ in Simulation A

eter $T$, making it convenient and weakly conservative to meet the temporal demands.

With regard to the transient process, the proposed PTESO exhibits high transient performance with trivial oscillation and a slight overshoot. However, the peaking values of $\tilde{x}_{2}$ and $\tilde{x}_{3}$ of the LESO, FTESO and FxTESO are very large, which may severely deteriorate the dynamic performance of the controller during the transient period of the ESO.

4.2 Simulation of the control system on a second-order system

In this subsection, the second-order system with mismatched and matched disturbances in Subsection 4.1

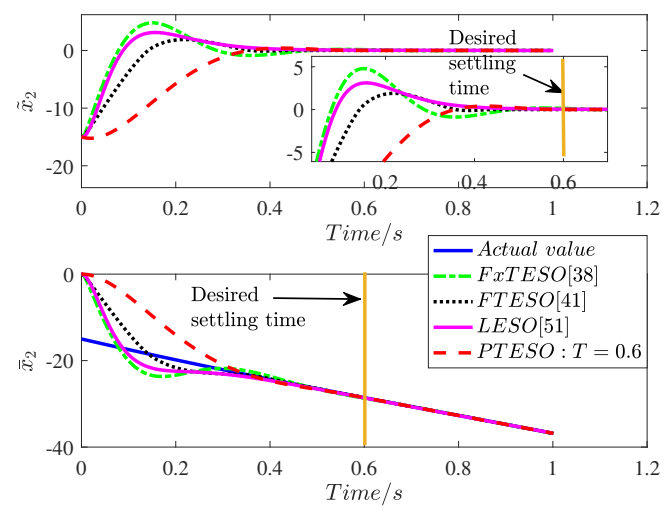

Fig. 6 Time histories of $\bar{x}_{2}$ and $\tilde{x}_{2}$ in Simulation B

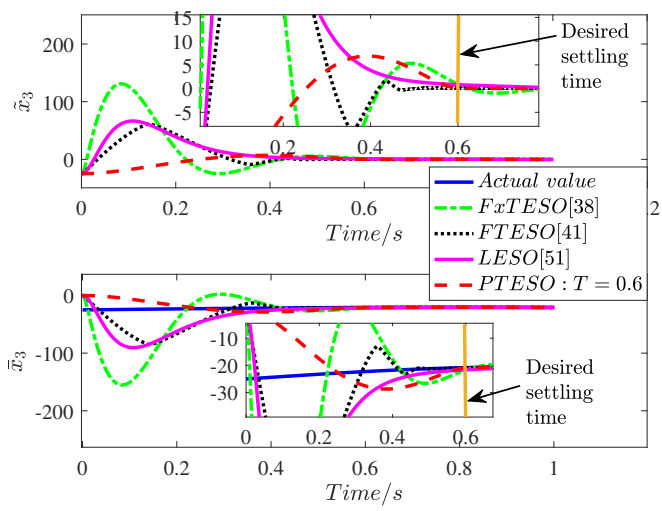

Fig. 7 Time histories of $\bar{x}_{3}$ and $\tilde{x}_{3}$ in Simulation B

is adopted to verify the efficiency of the proposed control system. For comparison, two existing PTSMC laws are introduced to be integrated with the PTESO (5). PTSMC law 1 is the method in [27] with the discontinuous term $\operatorname{sgn}(\cdot)$ substituted by $\hat{x}_{3}$. PTSMC law 2 utilizes the techniques in [14] and is depicted by:

$$
\begin{aligned}
& S_{1}=\hat{x}_{2}+\frac{1}{\left(1-p_{1}\right) \rho_{1}}\left\lceil x_{1}\right\rfloor^{p_{1}}\left(\left|x_{1}\right|+1\right)^{2-p_{1}} \\
& u=-\hat{x}_{3}-\frac{1}{\left(1-p_{1}\right) \rho_{2}}\left\lceil S_{1}\right\rfloor^{p_{1}}\left(\left|S_{1}\right|+1\right)^{2-p_{1}} \\
& -\frac{\hat{x}_{2}}{\left(1-p_{1}\right) \rho_{1}}\left[\begin{array}{c}
p_{1}\left|x_{1}\right|^{p_{1}-1}\left(\left|x_{1}\right|+1\right)^{2-p_{1}} \\
+\left(2-p_{1}\right)\left(\left|x_{1}\right|+1\right)^{1-p_{1}}\left|x_{1}\right|^{p_{1}}
\end{array}\right]
\end{aligned}
$$

where $\hat{x}_{2}$ and $\hat{x}_{3}$ are observed states from the PTESO (5). According to [14], by setting $\rho_{1}+\rho_{2}+T=t_{f 1}$, $x_{1}$ can be driven to the origin within the time interval $\left[t_{0}, t_{0}+t_{f 1}\right)$. Note that the three control laws in this simulation are continuous due to the introduction 
of PTESO (5). By this way, the PTSMC laws can be compared reasonably.

The initial time $t_{0}=0 \mathrm{~s} . x_{1}\left(t_{0}\right)=10, x_{2}\left(t_{0}\right)=5$. Simulation step size is 1e-3 s. Two groups of simulations are implemented with the prescribed UBST as $1 \mathrm{~s}$ in Group 1 and $1.5 \mathrm{~s}$ in Group 2. The parameter settings of PTESO remain the same as those in Simulation A of Subsection 4.1 except for $T=0.4$. The synthesis results of controllers are provided in Table 3. Since $u$ is unacceptably large at the beginning of the simulation case of PTSMC 2, we have to set the upper bound of $|u|$ as 5000. Simulation results are plotted in Figs. 8-13.

As shown in simulation results, much less conservativeness is exhibited by the proposed CPTSMC in meeting temporal demands than that of PTSMC 2. In two groups, $x_{1}$ under the drive of the CPTSMC converges to zero at approximately $t=t_{0}+t_{f 1}$. By contrast, PTSMC 2 drives $x_{1}$ to zero at an unnecessarily fast rate in Groups 1 and 2, in which $x_{1}$ reaches zero at approximately $t=0.5 \mathrm{~s}$ and $t=0.6 \mathrm{~s}$, respectively. Thus, the slack between the actual settling time and prescribed UBST of PTSMC 2 [14] is large. Moreover, $\dot{x}_{1}$ and $u$ in the case of PTSMC 2 become very large at the initial stage, while due to the high transient performance of the novel convergent form, $\dot{x}_{1}$ and $u$ in the case of CPTSMC change smoothly and have small peaking values. Therefore, the proposed CPTSMC is more desired for the fast convergence demands of practical applications under velocity constraints and saturation constraints, which are very common and can be found in $[49,50]$.

The advantages of the proposed CPTSMC over PTSMC 1 [27] are also indicated in Figs. 8-13. For the proposed CPTSMC, $x_{1}$ converges to zero in a mild manner. $x_{1}\left(t_{0}+t_{f 1}\right)=1.19 \mathrm{e}-6$ in Group 1 and $x_{1}\left(t_{0}+t_{f 1}\right)=4.38 \mathrm{e}-7$ in Group 2. Hence, the high convergence precision is maintained under the simulation step size 1e-3 s. However, for PTSMC $1, x_{1}$ converges to zero aggressively. $x_{1}\left(t_{0}+t_{f 1}\right)=-0.19$ and $x_{1}\left(t_{0}+t_{f 1}\right)=-0.017$ are realized in Groups 1 and 2 , respectively. In other words, a loss of precision occurs under the finite sampling frequency, which is in line with the analysis in Subsection 3.2.1. Moreover, $\dot{x}_{1}$ and $u$ at $t=t_{0}+t_{f 1}$ are large in the case of PTSMC 1 , owing to the precision loss. As a result, although $x_{1}$ driven by PTSMC 1 is near zero in Groups 1 and 2 at $t=t_{0}+t_{f 1}$, it escapes from the origin immediately. Therefore, it is concluded that the CPTSMC has a better transient performance and higher practical application value.
4.3 Simulation of a DC motor servo system under mismatched disturbances

To evaluate the practical effectiveness of the proposed control system, consider a DC servo motor with the state equations given by [34]:

$$
\left\{\begin{array}{l}
\dot{\omega}(t)=\frac{C_{m}}{J} i_{a}(t)-\frac{1}{J} M_{c}(t) \\
\dot{i}_{a}(t)=-\frac{C_{e}}{L_{a}} \omega(t)-\frac{R_{a}}{L_{a}} i_{a}(t)+\frac{1}{L_{a}} u(t)
\end{array}\right.
$$

where $L_{a}, i_{a}$, and $R_{a}$ denote the armature inductance, armature current, and resistance of the armature coil, respectively. $u$ is the applied voltage. $C_{e}$ is the electromotive force coefficient. $\omega, C_{m}$ and $J$ denote the rotational speed, torque constant, and inertia, respectively. $M_{c}$ represents the load disturbance torque.

As stated in [34], load disturbance torque $M_{c}$ and parameter perturbation are the main components of the lumped disturbances suffered by the motor servo system. The load disturbance torque $M_{c}$ refers to periodic oscillations of transmission components such as gear drive clearance, coupling clearance, and rolling mill roll eccentricity. The parameter perturbation includes the variation of mechanical parameter (e.g., the inertia $J$ ) and electromagnetic parameter (e.g., the resistance value of resistive elements, $R_{a}$ ). Since the variation of $J$ has a great influence on the system performance, we consider $J=J_{0}(1+\Delta J)$ in the simulation, where $J_{0}$ is the rotational inertia momentum of the servo motor and $J_{0} \Delta$ is the unknown load inertia momentum.

By defining the angular velocity command as $\omega_{r}$, the tracking errors $x_{1}=\omega_{r}-\omega$ and $x_{2}=\dot{\omega}_{r}-\dot{\omega}$ constitute the following system:

$\left\{\begin{array}{l}\dot{x}_{1}=x_{2} \\ \dot{x}_{2}=\frac{C_{m}}{J L_{a}}\left(\begin{array}{l}-u(t)+C_{e} \omega(t) \\ +R_{a} i_{a}(t)\end{array}\right)+\ddot{\omega}_{r}(t)+\frac{\dot{M}_{c}(t)}{J}\end{array}\right.$

which is within the framework of system (1). It is worth noting that $x_{2}$ is unmeasurable due to unknown mismatched disturbances caused by $M_{c}(t)$ and $\Delta J$.

In the simulation, $t_{0}=0 \mathrm{~s}, \omega\left(t_{0}\right)=0 \mathrm{rad} / \mathrm{s}$, $i_{a}\left(t_{0}\right)=0 \mathrm{~A}$, and $\omega_{r}(t)=\left(2 \cos \left(\frac{\pi t}{2}+\pi\right)+4\right) \mathrm{rad} / \mathrm{s}$. The following model parameters in [34] are adopted: $C_{e}=29 \mathrm{v} \cdot \mathrm{s} / \mathrm{rad}, C_{m}=29 \mathrm{~N} \cdot \mathrm{m} / \mathrm{A}, L_{a}=0.0003 \mathrm{H}$, $R_{a}=0.0314 \Omega, M_{c}=14500+300 \sin (3 \pi t) \mathrm{N} \cdot \mathrm{m}$, $J_{0}=1542 \mathrm{~kg} \cdot \mathrm{m}^{2}$, and $\Delta J=50 \mathrm{~kg} \cdot \mathrm{m}^{2}$. Four cases are simulated, in which the parameter $t_{f 1}$ is set as 1,2 , 3 , and 4 , respectively. Other parameter settings of the CPTSMC in four cases are: $\bar{a}=0.6$ and $\eta_{1}=2$. The synthesis results of PTESO in four cases are $T=0.4$, $\iota_{1}=0.1, \iota_{2}=0.3, \iota_{3}=0.09, \nu=1, k_{1}=3.823$, $k_{2}=6.945, k_{3}=1.5$, and $L=50$. The simulation results are provided in Figs. 15-18.

Time histories of the tracking error $x_{1}$ and observation errors $\left(\tilde{x}_{1}, \tilde{x}_{2}\right)$ are plotted in Figs. 15 and 18, 
Table 3 Parameter settings of PTSMC laws

\begin{tabular}{llll}
\hline Methods & CPTSMC & PTSMC 1 & PTSMC 2 \\
\hline Parameter settings (Group 1) & $\bar{a}=0.6, t_{f 1}=1, \eta_{1}=2$ & $t_{f 1}=1, \eta_{1}=2$ & $\rho_{1}=0.3, \rho_{2}=0.3, p_{1}=0.6$ \\
Parameter settings (Group 2) & $\bar{a}=0.6, t_{f 1}=1.5, \eta_{1}=2$ & $t_{f 1}=1.5, \eta_{1}=2$ & $\rho_{1}=0.55, \rho_{2}=0.55, p_{1}=0.6$ \\
\hline
\end{tabular}

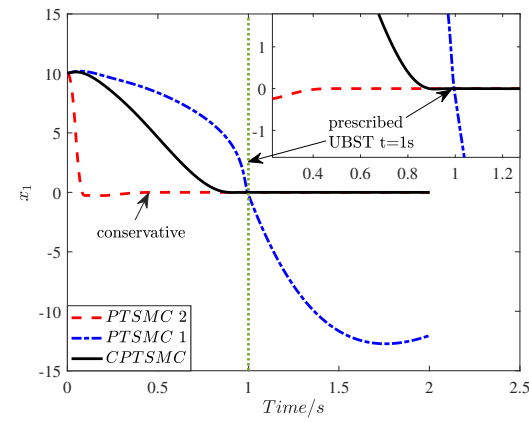

Fig. 8 Curves of $x_{1}$ in Group 1

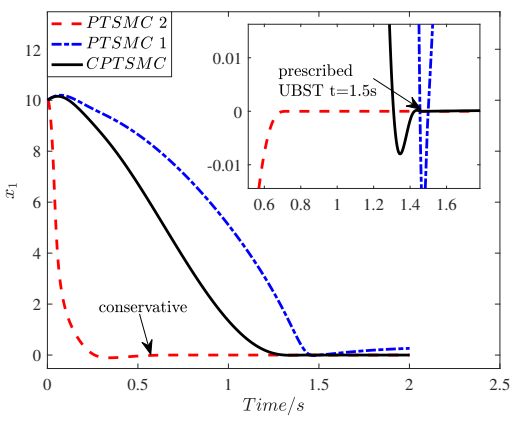

Fig. 11 Curves of $x_{1}$ in Group 2

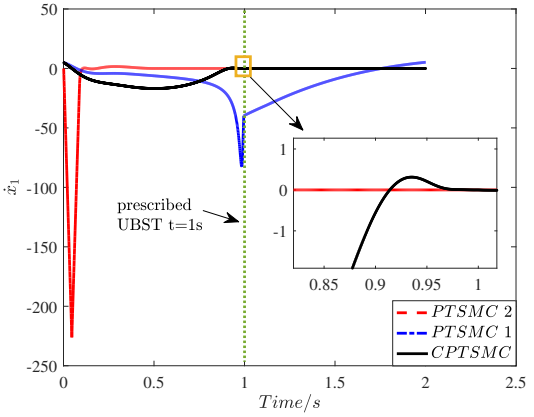

Fig. 9 Curves of $\dot{x}_{1}$ in Group 1

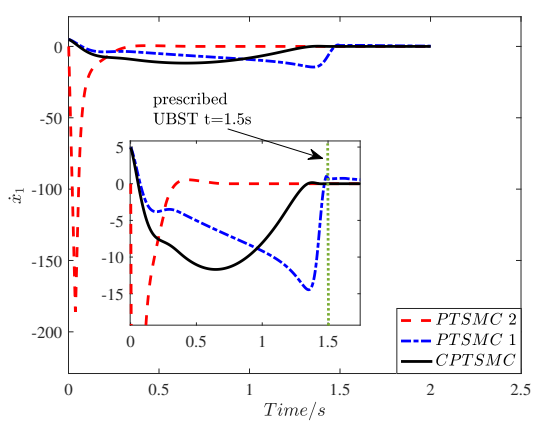

Fig. 12 Curves of $\dot{x}_{1}$ in Group 2

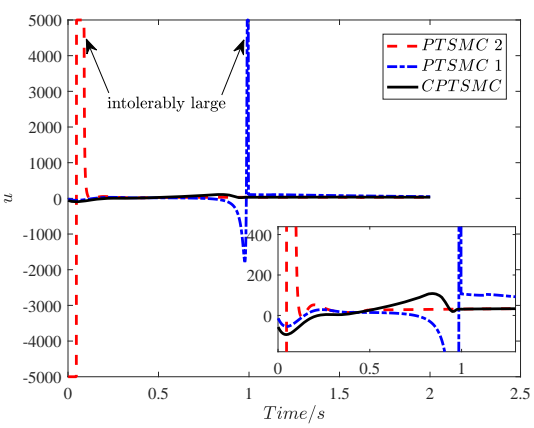

Fig. 10 Curves of $u$ in Group 1

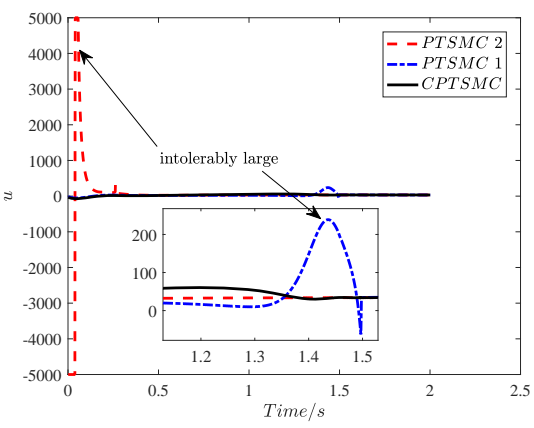

Fig. 13 Curves of $u$ in Group 2

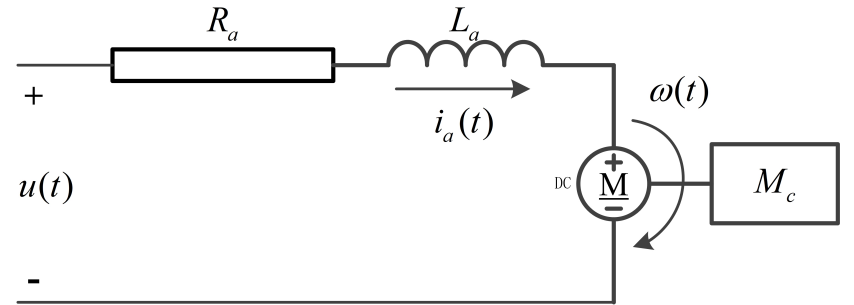

Fig. 14 Equivalent circuit diagram of DC servo motor

respectively. In four simulation cases, observation errors $\tilde{x}_{1}\left(t_{0}+T\right)=8.09 \mathrm{e}-8$ and $\tilde{x}_{2}\left(t_{0}+T\right)=-0.004$ are realized. Regarding the tracking error, $x_{1}\left(t_{0}+t_{f 1}\right)$ are $-0.0031,1.02 \mathrm{e}-5,0.0029$, and $2.8 \mathrm{e}-4$, respectively. Therefore, both the observation errors and tracking errors converge to zero within the prescribed time interval, validating the PTS of the proposed PTESO and CPTSMC. Moreover, as shown in Fig. 16, $\dot{x}_{1}$ also approaches zero near the corresponding switch time $t=t_{0}+t_{f 1} \cdot u(t), \omega(t)$ and $i_{a}(t)$ remain bounded and continuous during the simulation. Thus, the continuity of the CPTSMC and the boundedness of system signals are verified.

\section{Conclusion}

This paper addresses a prescribed-time control problem for a second-order nonlinear system with mismatched disturbance, which has not yet been solved. A PTESO is developed by employing a monotone increasing function over time. Precise estimation of the unknown system states and disturbances is realized with the UBST prescribed by one parameter. A weakly conservative and considerably simple tuning process for observation temporal demands is obtained. Moreover, due to the small initial values of correction terms, the peaking value problem has been well addressed. Then, a CPTSMC is developed by using the estimated states and disturbances of the PTESO. The controlled system states converge following the newly designed prescribed-time convergent form. The UBST of the system state is directly determined by one parameter of the CPTSMC. Like the PTESO, the CPTSMC also has a weakly conservative and simple tuning procedure for temporal demands. Furthermore, the boundedness of all system signals and the continuity of the control input have been validated. Numerical simulations are 


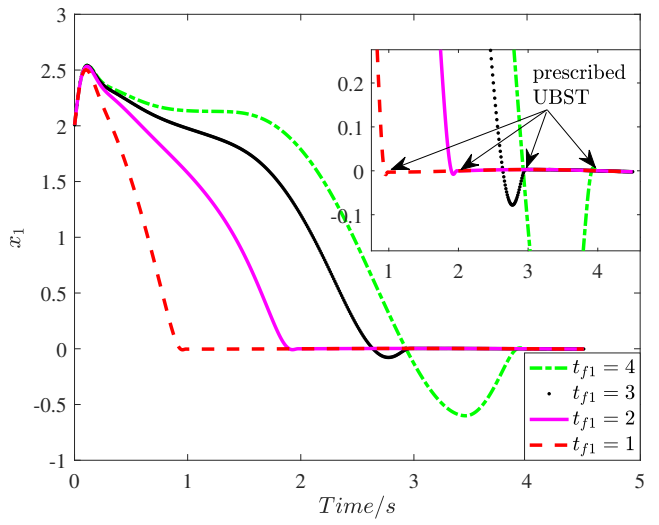

Fig. 15 Time histories of tracking errors
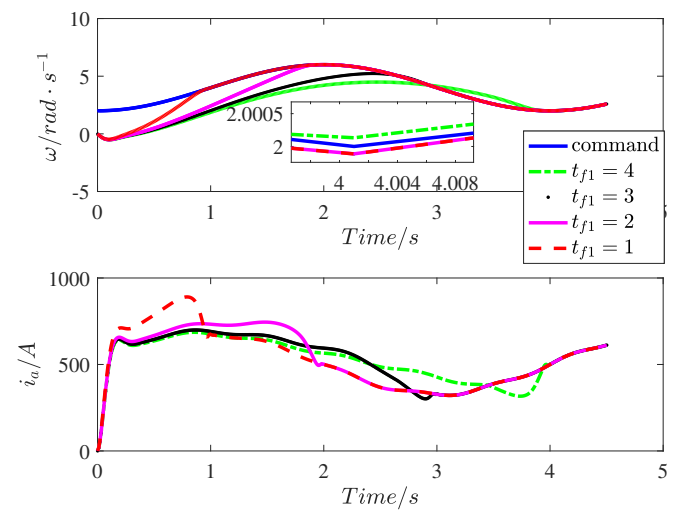

Fig. 17 Time histories of $\omega$ and $i_{a}$

implemented to verify the efficiency of the proposed PTESO and CPTSMC. The prescribed-time control for a high-order system with state-dependent and stateindependent mismatched disturbances will be explored in the future.

Acknowledgements This work was supported by the Chinese Scholarship Council under Grant No. 202006120134.

Data availability statement The data used to support the findings of this study are available from the author upon request.

\section{Declarations}

Conflict of interest All the authors of this manuscript declare that they have no conflict of interest.

\section{Appendix A Proof of Theorem 2}

The boundedness of the solution to Eq. (36) and uniform stability of the origin can be proved through the
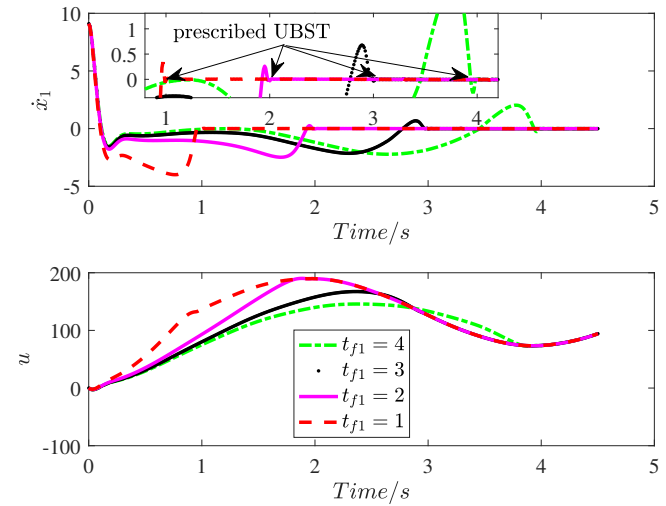

Fig. 16 Time histories of $\dot{x}_{1}$ and $u$

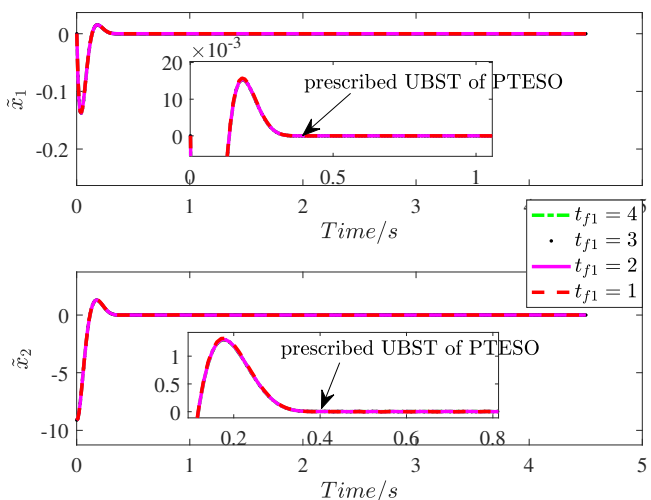

Fig. 18 Time histories of observation errors

deduction lines [27], which is omitted here for brevity. In this work, we focus on proving the PTS property. Let $\chi(t, \breve{\boldsymbol{x}})$ satisfy conditions i) and ii), and be the solution to the following differential equation with $\eta>1$ and $0<a \leq 1$ :

$\dot{\chi}=\frac{\left(e^{\chi^{a}}-1\right) \eta}{-a \chi^{a-1} e^{\chi^{a}}\left(t_{0}+t_{f}-t\right)}, \forall \chi \neq 0, \forall t \in I$

Further, transform Eq. (69) into:

$\frac{\mathrm{d} \chi}{\mathrm{d} t}=\frac{\left(e^{\chi^{a}}-1\right) \eta}{-a \chi^{a-1} e^{\chi^{a}}\left(t_{0}+t_{f}-t\right)}$

$\Rightarrow \frac{a \chi^{a-1} e^{\chi^{a}}}{\left(e^{\chi^{a}}-1\right)} \mathrm{d} \chi=\frac{-\eta}{t_{0}+t_{f}-t} \mathrm{~d} t$

Respectively, integrating the double sides of Eq. (70) yields:

$\int_{\chi\left(t_{0}\right)}^{\chi(t)} \frac{a \chi^{a-1} e^{\chi^{a}}}{e^{\chi^{a}-1}} \mathrm{~d} \chi=\int_{t_{0}}^{t} \frac{-\eta}{t_{0}+t_{f}-\tau} \mathrm{d} \tau$

$\left.\Rightarrow \ln \left(e^{\chi^{a}}-1\right)\right|_{\chi\left(t_{0}\right)} ^{\chi(t)}=\left.\ln \left(t_{0}+t_{f}-\tau\right)^{\eta}\right|_{t_{0}} ^{t}$

$\Rightarrow \ln \left(e^{\chi^{a}}-1\right)=\ln \left[\left(t_{0}+t_{f}-t\right)^{\eta} \Theta\right]$

where $\Theta=\frac{e^{\chi\left(t_{0}\right)^{a}}-1}{t_{f} \eta}$. Then the trajectories of $\chi(t)$ and $\dot{\chi}(t)$ can be given by:

$$
\begin{aligned}
& \chi(t)=\left[\ln \left(\left(t_{0}+t_{f}-t\right)^{\eta} \Theta+1\right)\right]^{\frac{1}{a}} \\
& \dot{\chi}(t)=\frac{\left\{\ln \left[\left(t_{0}+t_{f}-t\right)^{\eta} \Theta+1\right]\right\}^{\frac{1-a}{a}}}{a}\left[\frac{-\eta\left(t_{0}+t_{f}-t\right)^{\eta-1} \Theta}{\left(t_{0}+t_{f}-t\right)^{\eta} \Theta+1}\right]
\end{aligned}
$$


With $a \in(0,1]$ and $\eta>1, \chi\left(t_{0}+t_{f}\right)=0$ and $\dot{\chi}\left(t_{0}+t_{f}\right)=0$ are valid. The proof is complete.

\section{Appendix B Proof of Property 1}

According to Theorem 2, the solution to Eq. (36) can be expressed by Eq. (72). Through defining $\ell:=1 / a$ and $w:=|\dot{\chi}(t)|$, Eq. (72) is reshaped as $w=\zeta(\ell) U(\ell)$, where:

$$
\begin{aligned}
& \zeta(\ell)=\ell\left\{\ln \left[\left(t_{0}+t_{f}-t\right)^{\eta} \Theta+1\right]\right\}^{\ell-1} \\
& U(\ell)=\frac{\eta\left(t_{0}+t_{f}-t\right)^{\eta-1} \Theta}{\left(t_{0}+t_{f}-t\right)^{\eta} \Theta+1}
\end{aligned}
$$

Based on the chain derivation rule, the partial differential of $w$ with respect to $\ell$ is $\frac{\partial w}{\partial \ell}=\frac{\partial \zeta(\ell)}{\partial \ell} U(\ell)+$ $\zeta(\ell) \frac{\partial U(\ell)}{\partial \ell}$ with:

$$
\begin{aligned}
& \frac{\partial \zeta(\ell)}{\partial \ell}=\left\{\begin{array}{c}
1+\ell \ln \left\{\ln \left[\left(t_{0}+t_{f}-t\right)^{\eta} \Theta+1\right]\right\} \\
+\frac{\left(t_{0}+t_{f}-t\right)^{\eta}\left(\ell^{2}-\ell\right) \frac{\partial \Theta}{\partial \ell}}{\left[\left(t_{0}+t_{f}-t\right)^{\eta} \Theta+1\right] \ln \left[\left(t_{0}+t_{f}-t\right)^{\eta} \Theta+1\right]}
\end{array}\right\} \\
& \times\left\{\ln \left[\left(t_{0}+t_{f}-t\right)^{\eta} \Theta+1\right]\right\}^{\ell-1}
\end{aligned}
$$

$\frac{\partial U(\ell)}{\partial \ell}=\frac{\eta \frac{\partial \Theta}{\partial \ell}\left(t_{0}+t_{f}-t\right)^{\eta-1}}{\left[\left(t_{0}+t_{f}-t\right)^{\eta} \Theta+1\right]^{2}}$

if $a=1, \partial \zeta(\ell) / \partial \ell$ becomes negative as $t \rightarrow t_{0}+t_{f} \quad$ since $\ln \left\{\ln \left[\left(t_{0}+t_{f}-t\right)^{\eta} \Theta+1\right]\right\}$ tends to be negative infinity. if $a<1$, $\left(t_{0}+t_{f}-t\right)^{\eta} /\left\{\ln \left[\left(t_{0}+t_{f}-t\right)^{\eta} \Theta+1\right]\right\}$ tends to be a bounded constant as $t \rightarrow t_{0}+t_{f}$ according to L'Hôpital's rule [48], and $\ln \left\{\ln \left[\left(t_{0}+t_{f}-t\right)^{\eta} \Theta+1\right]\right\}$ becomes negatively infinite. Therefore, when $t$ is near $t_{0}+t_{f}$, there is:

$$
\begin{aligned}
& \frac{\partial \zeta(\ell)}{\partial \ell} \approx \ell\left\{\ln \left[\left(t_{0}+t_{f}-t\right)^{\eta} \Theta+1\right]\right\}^{\ell-1} \\
& \times \ln \left\{\ln \left[\left(t_{0}+t_{f}-t\right)^{\eta} \Theta+1\right]\right\}
\end{aligned}
$$

$$
\begin{aligned}
& \frac{\partial w}{\partial \ell} \approx\left\{\begin{array}{l}
\left.\left\{\ln \left[\left(t_{0}+t_{f}-t\right)^{\eta} \Theta+1\right]\right]\right\}^{\ell-1} \\
\times \ell \ln \left\{\ln \left[\left(t_{0}+t_{f}-t\right)^{\eta} \Theta+1\right]\right\} \frac{\eta\left(t_{0}+t_{f}-t\right)^{\eta-1} \Theta}{\left(t_{0}+t_{f}-t\right)^{\eta} \Theta+1}
\end{array}\right\} \\
& +\ell\left\{\ln \left[\left(t_{0}+t_{f}-t\right)^{\eta} \Theta+1\right]\right\}^{\ell-1} \frac{\eta \frac{\partial \Theta}{\partial \ell}\left(t_{0}+t_{f}-t\right)^{\eta-1}}{\left[\Theta\left(t_{0}+t_{f}-t\right)^{\eta}+1\right]^{2}} \\
& =\ell\left\{\ln \left[\left(t_{0}+t_{f}-t\right)^{\eta} \Theta+1\right]\right\}^{\ell-1} \frac{\eta\left(t_{0}+t_{f}-t\right)^{\eta-1}}{\Theta\left(t_{0}+t_{f}-t\right)^{\eta}+1} \\
& \times\left\{\Theta \ln \left\{\ln \left[\Theta\left(t_{0}+t_{f}-t\right)^{\eta}+1\right]\right\}+\frac{\partial \Theta / \partial \ell}{\left(t_{0}+t_{f}-t\right)^{\eta} \Theta+1}\right\}
\end{aligned}
$$

Since $\partial \Theta / \partial \ell$ is bounded and $\ln \left\{\ln \left[\left(t_{0}+t_{f}-t\right)^{\eta} \Theta+1\right]\right\} \quad$ becomes negative infinity as $t \rightarrow t_{0}+t_{f}, \partial w / \partial \ell$ turns negative as $t \rightarrow t_{0}+t_{f}$. In other words, $\partial w / \partial a$ turns positive as $t \rightarrow t_{0}+t_{f}$. As a result, there always exists a time interval $\left[t_{0}+t_{f}-m, t_{0}+t_{f}\right)$ with $m>0$, such that for $t \in\left[t_{0}+t_{f}-m, t_{0}+t_{f}\right)$, the solution to Eq. (36) decreases with decreasing $a$. This completes the proof of Property 1.

\section{References}

1. Sheng, Y., Zhang, Z., Xia, L. Fractional-order sliding mode control based guidance law with impact angle constraint. Nonlinear Dyn. (2021). Doi: 10.1007/s11071-02106820-6.

2. Biswas B, Maity A, Kumar S R. Finite-time convergent three-dimensional nonlinear intercept angle guidance. J Guid Control Dyn, 43(1): 146-153 (2020).

3. Li, P., Boem, F., Pin, G. Fast-convergent fault detection and isolation in a class of nonlinear uncertain systems. Eur J Control, 55: 45-55, (2020).

4. Xia, K., Zou, Y. Adaptive fixed-time fault-tolerant control for noncooperative spacecraft proximity using relative motion information. Nonlinear Dyn, 100(3), 2521-2535, 2020.

5. Jiang, B., Li, C., Hou, S., Ma, G. Fixed-time attitude tracking control for spacecraft based on adding power integrator technique. Int J Robust Nonlinear Control, 30(6): 2515-2532 (2020).

6. Du, H., Zhang, J., Wu, D., Zhu, W., Li, H., Chu, Z. Fixedtime attitude stabilization for a rigid spacecraft. ISA Trans, 98: 263-270 (2020).

7. Song, G., Shi, P., Agarwal, R. K. Fixed-time sliding mode cooperative control for multiagent networks via eventtriggered strategy. Int J Robust Nonlinear Control, 31(1): 21-36 (2021).

8. Gong, K., Liao, Y., Wang, Y. Adaptive fixed-time terminal sliding mode control on SE (3) for coupled spacecraft tracking maneuver. Int J Aerospace Eng. (2020). Doi: $10.1155 / 2020 / 3085495$.

9. Wang, L., Du, H., Zhang, W. et al. Implementation of integral fixed-time sliding mode controller for speed regulation of PMSM servo system. Nonlinear Dyn. 102: 185-196 (2020).

10. Abadi, A. S. S., Hosseinabadi, P. A., Mekhilef, S. (2020). Fuzzy adaptive fixed-time sliding mode control with state observer for a class of high-order mismatched uncertain systems. Int J Control Autom, 18: 2492-2508 (2021).

11. Cruz-Zavala, E., Moreno, J. A. High-order sliding-mode control design homogeneous in the bi-limit. Int J Robust Nonlinear Control, 31(9): 3380-3416 (2021).

12. Bernuau, E., Efimov, D., Perruquetti, W., Polyakov, A. On homogeneity and its application in sliding mode control. J. Franklin Inst., 351(4): 1866-1901 (2014).

13. Gómez-Gutiérrez, D. On the design of nonautonomous fixed-time controllers with a predefined upper bound of the settling time. Int J Robust Nonlinear Control, 30(10): 38713885 (2020).

14. Jiménez-Rodríguez, E., Muñoz-Vázquez, A. J., SánchezTorres, J. D., Defoort, M., Loukianov, A. G. A Lyapunovlike characterization of predefined-time stability. IEEE Trans. Autom. Control, 65(11): 4922-4927 (2020).

15. Liang, C. D., Ge, M. F., Liu, Z. W., Ling, G., Zhao, X. W. A novel sliding surface design for predefined-time stabilization of Euler-Lagrange systems. Nonlinear Dyn, (2021). Doi: 10.1007/s11071-021-06826-0.

16. Muñoz-Vázquez, A. J., Fernández-Anaya, G., SánchezTorres, J. D., Meléndez-Vázquez, F. Predefined-time control of distributed-order systems. Nonlinear Dyn, 103(3): 2689-2700 (2021).

17. Wang, Y., Wang, Z., Chen, M., Kong, L. Predefined-time sliding mode formation control for multiple autonomous underwater vehicles with uncertainties. Chaos, Solitons Fractals, 144: 110680 (2021).

18. Liang, C. D., Ge, M. F., Liu, Z. W., Wang, Y. W., Karimi, H. R. Predefined-time Terminal Sliding 
Mode Control of Robot Manipulators. arXiv preprint arXiv:2001.04198 (2020)

19. Becerra, H. M., Vázquez, C. R., Arechavaleta, G., Delfin, J. Predefined-time convergence control for high-order integrator systems using time base generators. IEEE Trans Control Syst Technol, 26(5): 1866-1873 (2017).

20. Gómez-Gutiérrez, D. On the design of nonautonomous fixed-time controllers with a predefined upper bound of the settling time. Int J Robust Nonlinear Control, 30(10): 38713885 (2020).

21. Ding, C., Shi, C., Chen, Y. Nonsingular prescribed-time stabilization of a class of uncertain nonlinear systems: A novel coordinate mapping method. Int J Robust Nonlinear Control, 30(9): 3566-3581 (2020).

22. Zhou, B., Shi, Y. Prescribed-time stabilization of a class of nonlinear systems by linear time-varying feedback. IEEE Trans. Autom. Control (2021). Doi: 10.1109/TAC.2021.3061645.

23. Krishnamurthy, P., Khorrami, F., Krstic, M. Robust adaptive prescribed-time stabilization via output feedback for uncertain nonlinear strict-feedback-like systems. Eur. J. Control, 55: 14-23 (2020)

24. Li, W., Krstic, M. Stochastic nonlinear prescribed-time stabilization and inverse optimality. IEEE Trans. Autom. Control (2021). Doi: 10.1109/TAC.2021.3061646.

25. Holloway, J., Krstic, M. Prescribed-time output feedback for linear systems in controllable canonical form. Automatica, 107: 77-85 (2019).

26. Song, Y., Wang, Y., Holloway, J., Krstic, M. Timevarying feedback for regulation of normal-form nonlinear systems in prescribed finite time. Automatica, 83: 243-251 (2017).

27. Pal, A. K., Kamal, S., Nagar, S. K., Bandyopadhyay, B., Fridman, L. Design of controllers with arbitrary convergence time. Automatica, 112: 108710 (2020).

28. Pal, A. K., Kamal, S., Nagar, S. K., Byopadhyay, B., Fridman, L. Authors' Reply To:(CI 20-0229) Comments on Design of controllers with arbitrary convergence time $[\mathrm{Au}-$ tomatica 108710] (2020)

29. Cui, L., Jin, N. Prescribed-time ESO-based prescribedtime control and its application to partial IGC design. Nonlinear Dyn, 1-18 (2021). Doi: 10.1007/s11071-021-06859-5.

30. Chen, Z., Ju, X., Wang, Z., Li, Q. The prescribed time sliding mode control for attitude tracking of spacecraft. Asian J Control (2021). Doi: 10.1002/asjc.2569.

31. Sheng, Y., Bai, W., Xie, Y. Fractional-order $P I^{\lambda} D$ sliding mode control for hypersonic vehicles with neural network disturbance compensator. Nonlinear Dyn, 103(1): 849863 (2021).

32. Qin, W., Ge, P., Liu, F., Long, S. Adaptive robust control for active suspension systems: targeting nonholonomic reference trajectory and large mismatched uncertainty. Nonlinear Dyn, 104: 3861-3880 (2021).

33. Liang, X., Wang, Q., Hu, C., Dong, C. Fixed-time observer based fault-tolerant attitude control for reusable launch vehicle with actuator faults. Aerosp Sci Technol, 107: 106314 (2020).

34. Zhou, L., Jiang, F., She, J., Zhang, Z. Generalizedextended-state-observer-based repetitive control for DC motor servo system with mismatched disturbances. Int J Control Autom, 1-10 (2020).

35. Wang, J., Li, S., Yang, J., Wu, B., Li, Q. Finite-time disturbance observer based non-singular terminal slidingmode control for pulse width modulation based DC-DC buck converters with mismatched load disturbances. IET Power Electron., 9(9): 1995-2002 (2016).
36. Wu, Z. H., Guo, B. Z. Approximate decoupling and output tracking for MIMO nonlinear systems with mismatched uncertainties via ADRC approach. J. Franklin Inst., 355(9): 3873-3894 (2018).

37. Zhao, Z. L., Guo, B. Z. A novel extended state observer for output tracking of MIMO systems with mismatched uncertainty. IEEE Trans. Autom. Control, 63(1): 211-218 (2017).

38. Cui, L., Jin, N., Chang, S., Zuo, Z., Zhao, Z. Fixedtime ESO based fixed-time integral terminal sliding mode controller design for a missile. ISA Trans (2021). Doi: 10.1016/j.isatra.2021.06.039.

39. Chang, S., Wang, Y., Zuo, Z. Fixed-Time Active Disturbance Rejection Control and Its Application to Wheeled Mobile Robots. IEEE Trans. Syst. Man Cybern. Syst. (2020). Doi: 10.1109/TSMC.2020.2966077.

40. Liu, J., Sun, M., Chen, Z., Sun, Q. Super-twisting sliding mode control for aircraft at high angle of attack based on finite-time extended state observer. Nonlinear Dyn, 99(4): 2785-2799 (2020).

41. Basin, M. Finite-and fixed-time convergent algorithms: Design and convergence time estimation. Annu Rev Control, 48: 209-221 (2019).

42. Aldana-López, R., Gómez-Gutiérrez, D., Trujillo, M. A., Navarro-Gutiérrez, M., Ruiz-León, J., Becerra, H. M. A predefined-time first-order exact differentiator based on time-varying gains. Int J Robust Nonlinear Control (2021). Doi: $10.1002 /$ rnc.5536.

43. Seeber, R., Haimovich, H., Horn, M., Fridman, L. M., De Battista, H. Robust exact differentiators with predefined convergence time. Automatica, 134: 109858 (2021).

44. Ni, J., Liu, L., Tang, Y., Liu, C. Predefined-time consensus tracking of second-order multiagent systems. IEEE Trans. Syst. Man Cybern. Syst., 51(4): 2550-2560, (2021).

45. Holloway, J., Krstic, M. Prescribed-time observers for linear systems in observer canonical form. IEEE Trans. Autom. Control, 64(9): 3905-3912 (2019).

46. Ginoya, D., Shendge, P. D., Phadke, S. B. Sliding mode control for mismatched uncertain systems using an extended disturbance observer. IEEE Trans. Ind. Electron., 61(4): 1983-1992 (2013).

47. Cruz-Zavala, E., Moreno, J. A. Levant's arbitrary-order exact differentiator: a Lyapunov approach. IEEE Trans. Autom. Control, 64(7): 3034-3039 (2018).

48. Wang, Z., Liang, B., Sun, Y., Zhang, T. Adaptive faulttolerant prescribed-time control for teleoperation systems with position error constraints. IEEE Trans Industr Inform, 16(7): 4889-4899 (2019).

49. Chen, H., Liu, Y. J., Liu, L., Tong, S., Gao, Z. AntiSaturation-Based Adaptive Sliding-Mode Control for Active Suspension Systems With Time-Varying Vertical Displacement and Speed Constraints. IEEE Trans Cybern, (2021). Doi: 10.1109/TCYB.2020.3042613.

50. Qiao, H. Y., Meng, H., Wang, M. J., Ke, W., Sun, J. G. Adaptive control for hypersonic vehicle with input saturation and state constraints. Aerosp Sci Technol, 84: 107-119 (2019).

51. Li, P., Wang, L., Zhu, G., Zhang, M. Predictive active disturbance rejection control for servo systems with communication delays via sliding mode approach. IEEE Trans. Ind. Electron., 68(12): 12679-12688 (2020). 